\title{
NONLINEAR NONOVERLAPPING SCHWARZ WAVEFORM RELAXATION FOR SEMILINEAR WAVE PROPAGATION
}

\author{
LAURENCE HALPERN AND JÉRÉMIE SZEFTEL
}

\begin{abstract}
We introduce a nonoverlapping variant of the Schwarz waveform relaxation algorithm for semilinear wave propagation in one dimension. Using the theory of absorbing boundary conditions, we derive a new nonlinear algorithm. We show that the algorithm is well-posed and we prove its convergence by energy estimates and a Galerkin method. We then introduce an explicit scheme. We prove the convergence of the discrete algorithm with suitable assumptions on the nonlinearity. We finally illustrate our analysis with numerical experiments.
\end{abstract}

\section{INTRODUCTION}

Schwarz waveform relaxation is a new class of algorithms for domain decomposition in the frame of time dependent partial differential equations. They are well-adapted to evolution problems, designed to solve the equations separately on each spatial subdomain on the whole time interval, exchanging information on the space-time boundary of the subdomains, overlapping or not [6].

In particular, for the computation of wave propagation, it is of great importance, due to numerical dispersion, to be able to handle local time and space meshes, and this is allowed by these new algorithms. We presented the method for the linear wave equation in [7] and 5. When using overlapping subdomains and "classical" Schwarz waveform relaxation (by a Dirichlet exchange of information on the boundary) the so-defined algorithm converges in a finite number of iterations, inversely proportional to the size of the overlap, which increases the storage and computational load. We therefore introduced optimized transmission conditions, relying on the theory of absorbing boundary conditions, which improved drastically the convergence of the algorithm.

This paper is a first attempt to extend the strategy to nonlinear equations. An analysis of the classical Schwarz waveform relaxation algorithm for convection dominated nonlinear conservation laws was performed in [4, but no other transmission conditions have been used so far. We are interested here in the semilinear wave equation. This equation intervenes in various phenomena as the dislocation in crystals, laser pulses in plasmas, etc. (see for instance [13]). In the latter example, as

Received by the editor January 31, 2007 and, in revised form, March 27, 2008.

2000 Mathematics Subject Classification. Primary 65F10, 65N22.

Key words and phrases. Domain decomposition, waveform relaxation, Schwarz methods, semilinear wave equation.

The second author was partially supported by NSF Grant DMS-0504720.

(C)2008 American Mathematical Society Reverts to public domain 28 years from publication 
the laser wavelength and the Debye length of the plasma can differ by several orders of magnitude, discretizations sufficiently fine to resolve the short scales yield systems of equations far beyond the power of current computers. By using domain decomposition techniques, decoupling different time scales becomes possible.

As a first step, the goal of this paper is to define new Schwarz waveform relaxation algorithms for the semilinear wave equation. We introduce two nonoverlapping algorithms. The first one referred to as linear uses the absorbing boundary condition of the linear problem, whereas the second one referred to as nonlinear uses the nonlinear absorbing boundary conditions designed by J. Szeftel in 12. The paper is organized as follows.

In Section 2 we introduce the definitions of the algorithms.

In Section 3 we prove the algorithms to be well-posed. For the precise analysis, we use a fixed point algorithm with regularity estimates on a linear problem.

In Section 4 we prove the convergence of the algorithms. The proof is an extension of a clever trick in [8], already used for linear algorithms, either hyperbolic or parabolic (see [7]). However, the nonlinearity requires a very fine analysis.

In Section 5 we design discrete Schwarz waveform relaxation algorithms. In each subdomain, the interior scheme is the usual leapfrog scheme for the linear part, with a downwinding in time for the nonlinear part. The exchange of information on the boundary is naturally taken into account by a finite volume strategy. In Section 6 we study the convergence of the algorithms by discrete energy estimates.

As it is always the case for nonlinear problems, the well-posedness and convergence results hold only locally in time. Therefore numerical experiments are very important to bypass the limitations of the theory. We present the results in Section 7, showing in particular that our nonlinear algorithm gives optimal results within a large class of algorithms.

Remark. Due to the complexity of the mathematical theory, we restrain ourselves to the one-dimensional case. The multidimensional study contains additional difficulties due to the geometry and should be considered in a forthcoming paper.

\section{Problem DESCRIPTION}

We consider the second order semilinear wave equation in one dimension,

$$
\left(\partial_{t}^{2}-\partial_{x}^{2}\right) \mathcal{U}=f\left(\mathcal{U}, \partial_{t} \mathcal{U}, \partial_{x} \mathcal{U}\right)
$$

on the domain $\mathbb{R} \times(0, T)$ with initial conditions $\mathcal{U}(\cdot, 0)=p, \partial_{t} \mathcal{U}(\cdot, 0)=q$.

2.1. Absorbing boundary conditions for the semilinear wave equation. The question of absorbing boundary conditions arises when one wants to make computations on an unbounded domain: a bounded computational domain is introduced, on the boundary of which boundary conditions must be prescribed. These boundary conditions must be absorbing to the waves leaving the domain. A whole strategy has been designed by Engquist and Majda for linear problems with variable coefficients, using pseudo-differential operators [3]. Recently it has been extended to nonlinear operators by J. Szeftel, in particular, for the semilinear wave equation [12, using the paradifferential calculus of [1] and [9]. We introduce a family of operators

$$
\mathcal{B}^{ \pm}\left(g^{ \pm}\right) u=\partial_{t} u \pm \partial_{x} u+g^{ \pm}(u)
$$


for $C^{\infty}$ functions $g^{ \pm}$such that $g^{ \pm}(0)=0$. The linear absorbing boundary operators are given by $g^{ \pm}=0$. In the case where $f\left(u, u_{t}, u_{x}\right)=f_{1}(u)+f_{2}(u) u_{t}+f_{3}(u) u_{x}$ with $f_{j}$ in $C^{\infty}(\mathbb{R}), 1 \leq j \leq 3$, and $f_{1}(0)=0$, the following nonlinear boundary operators are given in [12]:

$$
g^{+}(u):=-\frac{1}{2} \int_{0}^{u}\left(f_{2}-f_{3}\right)(\xi) d \xi, \quad g^{-}(u):=-\frac{1}{2} \int_{0}^{u}\left(f_{2}+f_{3}\right)(\xi) d \xi
$$

We replace the problem on the domain $\mathbb{R}$ by a boundary value problem in $\Omega_{0}=$ $(a, b)$ :

$$
\begin{gathered}
\left(\partial_{t}^{2}-\partial_{x}^{2}\right) \bar{u}=f\left(\bar{u}, \partial_{t} \bar{u}, \partial_{x} \bar{u}\right) \text { in } \Omega_{0} \times(0, T), \\
\mathcal{B}^{-}\left(g^{-}\right) \bar{u}(a, \cdot)=0, \quad \mathcal{B}^{+}\left(g^{+}\right) \bar{u}(b, \cdot)=0,
\end{gathered}
$$

with initial values $p$ and $q$. Such boundary conditions give well-posed initial boundary value problems, and are absorbing provided the intial data is compactly supported in $\Omega_{0}$; see [12. Following the strategy in [7, we use such absorbing operators for domain decomposition.

\subsection{A general nonoverlapping Schwarz waveform relaxation algorithm.}

We decompose the domain $(a, b)$ into $I$ nonoverlapping subdomains $\Omega_{i}=\left(a_{i}, a_{i+1}\right)$, $a_{j}<a_{i}$ for $j<i$ and $a_{1}=a, a_{I+1}=b$, and we introduce a general nonoverlapping Schwarz waveform relaxation algorithm. An initial guess $\left\{h_{i}^{ \pm, 0}\right\}_{1 \leq i \leq I+1}$ is given. For $k \geq 1$, one step of the algorithm is

$$
\begin{aligned}
& {\left[\left(\partial_{t}^{2}-\partial_{x}^{2}\right) u_{i}^{k}=f\left(u_{i}^{k}, \partial_{t} u_{i}^{k}, \partial_{x} u_{i}^{k}\right) \text { in } \Omega_{i} \times(0, T),\right.} \\
& u_{i}^{k}(\cdot, 0)=p, \quad \partial_{t} u_{i}^{k}(\cdot, 0)=q \text { in } \Omega_{i} \text {, } \\
& \mathcal{B}^{-}\left(g^{-}\right) u_{i}^{k}\left(a_{i}, \cdot\right)=h_{i}^{-, k-1}, \quad \mathcal{B}^{+}\left(g^{+}\right) u_{i}^{k}\left(a_{i+1}, \cdot\right)=h_{i}^{+, k-1} \text { in }(0, T) \text {, } \\
& h_{i}^{-, k}=\mathcal{B}^{-}\left(g^{-}\right) u_{i-1}^{k}\left(a_{i}, \cdot\right), \quad h_{i}^{+, k}=\mathcal{B}^{+}\left(g^{+}\right) u_{i+1}^{k}\left(a_{i+1}, \cdot\right) \text { in }(0, T),
\end{aligned}
$$

where $\mathcal{B}^{ \pm}\left(g^{ \pm}\right)$are given in (2.2). For ease of notation, we defined here $h_{1}^{ \pm, k}=0$ and $h_{I+1}^{ \pm, k}=0$, so that the index $i$ in (2.5) ranges from $i=1,2, \ldots, I$. In the sequel, we call linear transmission condition the choice $g^{ \pm}=0$ and nonlinear transmission condition the choice (2.3). For the classical linear homogeneous wave equation, it has been proved in [7] that the algorithm converges optimally if $T$ is small enough (which means in two iterations, independently of the number of subdomains), and the transmission operators $\mathcal{B}^{ \pm}$are given by $\mathcal{B}^{ \pm}=\partial_{t} \pm \partial_{x}$. This behavior is due to the finite speed of propagation, together with the fact that these operators are the exact Dirichlet-Neumann operators in this case. In the nonlinear case, the propagation still takes place with the finite speed, but we can use only approximate Dirichlet Neumann operators. Therefore the classical Schwarz algorithm with overlap is still convergent, and for our nonoverlapping nonlinear algorithms, we will use energy estimates.

\section{WELL-POSEDNESS FOR THE SUBPROBLEMS}

The study of the nonlinear problem relies on an iterative linear scheme. Therefore a first step for the definition of the algorithm is to study the nonhomogeneous initial boundary value problem for a general domain $\Omega=\left(a_{-}, a_{+}\right)$,

$$
\begin{gathered}
\left(\partial_{t}^{2}-\partial_{x}^{2}\right) u=f\left(u, \partial_{t} u, \partial_{x} u\right) \text { in } \Omega \times(0, T), \\
\mathcal{B}^{-}\left(g^{-}\right) u\left(a_{-}, \cdot\right)=h^{-}, \quad \mathcal{B}^{+}\left(g^{+}\right) u\left(a_{+}, \cdot\right)=h^{+},
\end{gathered}
$$


with initial values $p$ and $q$. We will use for $j \leq 2$ the spaces

$$
V_{j}(\Omega, T)=\left\{u \in L^{\infty}\left(0, T ; L^{2}(\Omega)\right), \partial^{\alpha} u \in L^{\infty}\left(0, T ; L^{2}(\Omega)\right),|\alpha| \leq j\right\} .
$$

In formula (3.2), $\alpha$ is a 2-index in $\mathbb{N}^{2}$, the first coordinate in $\alpha$ stands for the time, and the second one stands for the space, so for instance $\partial^{\alpha} u=\partial_{t x} u$ for $\alpha=(1,1)$. $V_{j}(\Omega, T)$ is equipped with the norm $\|u\|_{V_{j}(\Omega, T)}=\max _{|\alpha| \leq j}\left\|\partial^{\alpha} u\right\|_{L^{\infty}\left(0, T ; L^{2}(\Omega)\right)}$.

Theorem 3.1. Let $p$ be in $H^{2}(\Omega)$ and $q$ be in $H^{1}(\Omega)$. There exists a time $T^{*}$ such that for any $T \leq T^{*}$, for $h^{ \pm}$in $H^{1}(0, T)$ with the compatibility conditions

$$
h^{ \pm}(0)=q\left(a_{ \pm}\right) \pm p^{\prime}\left(a_{ \pm}\right)+g^{ \pm}\left(p\left(a_{ \pm}\right)\right),
$$

(3.1) has a unique solution $u$ in $V_{2}(\Omega, T)$, with $\partial_{t} u\left(a_{ \pm}, \cdot\right)$ and $\partial_{x} u\left(a_{ \pm}, \cdot\right)$ in $H^{1}(0, T)$. Furthermore, there exists a positive real number $C^{*}$ such that

$$
\begin{aligned}
& \|u\|_{V_{2}(\Omega, T)}^{2}+\sum_{ \pm} \sum_{|\alpha|=1}\left\|\partial^{\alpha} u\left(a_{ \pm}, \cdot\right)\right\|_{H^{1}(0, T)}^{2} \\
& \quad \leq C^{*}\left(\|p\|_{H^{2}(\Omega)}^{2}+\|q\|_{H^{1}(\Omega)}^{2}+\sum_{ \pm}\left\|h^{ \pm}\right\|_{H^{1}(0, T)}^{2}\right),
\end{aligned}
$$

where $T^{*}$ and $C^{*}$ depend on the data $p, q, f, g^{ \pm}, h^{ \pm}$.

This result was first proved in [12] with homogeneous boundary conditions (i.e. $h^{ \pm}=0$ ). The additional difficulty comes from the boundary conditions, and we give here the main steps of the proof. It relies on the construction of a sequence of linear problems of the form

$$
\begin{gathered}
\partial_{t}^{2} \tilde{u}-\partial_{x}^{2} \tilde{u}+\tilde{u}=F \text { in } \Omega \times(0, T), \\
\left(\partial_{t} \tilde{u}-\partial_{x} \tilde{u}\right)\left(a_{-}, \cdot\right)=H^{-}, \quad\left(\partial_{t} \tilde{u}+\partial_{x} \tilde{u}\right)\left(a_{+}, \cdot\right)=H^{+} .
\end{gathered}
$$

Proposition 3.2. Let $p$ be in $H^{2}(\Omega)$ and $q$ be in $H^{1}(\Omega)$. For any positive time $T$, let $F$ be in $H^{1}((0, T) \times \Omega)$, and $H^{ \pm}$be in $H^{1}(0, T)$ with the compatibility conditions

$$
H^{ \pm}(0)=q\left(a_{ \pm}\right) \pm p^{\prime}\left(a_{ \pm}\right) \text {. }
$$

Then, (3.5) with initial data $p$ and $q$ has a unique solution $\tilde{u}$ in $V_{2}(\Omega, T)$, with $\partial_{t} \tilde{u}\left(a_{ \pm}, \cdot\right)$ and $\partial_{x} \tilde{u}\left(a_{ \pm}, \cdot\right)$ in $H^{1}(0, T)$. Moreover, we have the following bounds on the solution

$$
\begin{gathered}
\|\tilde{u}\|_{V_{2}(\Omega, T)}^{2}+\sum_{ \pm}\left(\left\|\partial_{t} \tilde{u}\left(a_{ \pm}, \cdot\right)\right\|_{H^{1}(0, T)}^{2}+\left\|\partial_{x} \tilde{u}\left(a_{ \pm}, \cdot\right)\right\|_{H^{1}(0, T)}^{2}\right) \\
\leq C_{1} e^{T}\left(\|F\|_{H^{1}\left(0, T ; L^{2}(\Omega)\right)}^{2}+\|F(\cdot, 0)\|_{L^{2}(\Omega)}^{2}\right. \\
\left.\quad+\sum_{ \pm}\left\|H^{ \pm}\right\|_{H^{1}(0, T)}^{2}+\|p\|_{H^{2}(\Omega)}^{2}+\|q\|_{H^{1}(\Omega)}^{2}\right)
\end{gathered}
$$

where $C_{1}$ is a universal constant.

Proof. We start with the a priori estimates. We multiply (3.5) by $\partial_{t} \tilde{u}$ and integrate by parts in $\Omega$ :

$$
\begin{aligned}
\frac{1}{2} \frac{d}{d t}\left(\left\|\partial_{t} \tilde{u}(\cdot, t)\right\|_{L^{2}(\Omega)}^{2}+\|\tilde{u}(\cdot, t)\|_{H^{1}(\Omega)}^{2}\right)+\left(\partial_{t} \tilde{u}\left(a_{-}, t\right)\right)^{2}+\left(\partial_{t} \tilde{u}\left(a_{+}, t\right)\right)^{2} \\
=\left(F(\cdot, t), \partial_{t} \tilde{u}(\cdot, t)\right)_{L^{2}(\Omega)}+H^{-}(t) \partial_{t} \tilde{u}\left(a_{-}, t\right)+H^{+}(t) \partial_{t} \tilde{u}\left(a_{+}, t\right) .
\end{aligned}
$$


Using the Cauchy-Schwarz inequality on the right-hand side, together with the inequality $\alpha \beta \leq \frac{1}{2} \alpha^{2}+\frac{1}{2} \beta^{2}$ for all $\alpha, \beta \in \mathbb{R}$, and finally integrating in time, we obtain

$$
\begin{aligned}
\left\|\partial_{t} \tilde{u}(\cdot, t)\right\|_{L^{2}(\Omega)}^{2}+ & \|\tilde{u}(\cdot, t)\|_{H^{1}(\Omega)}^{2}+\int_{0}^{t}\left[\left(\partial_{t} \tilde{u}\left(a_{-}, s\right)\right)^{2}+\left(\partial_{t} \tilde{u}\left(a_{+}, s\right)\right)^{2}\right] d s \\
\leq & \int_{0}^{t}\left[\left\|\partial_{t} \tilde{u}(\cdot, s)\right\|_{L^{2}(\Omega)}^{2} d s+\int_{0}^{t}\|F(\cdot, s)\|_{L^{2}(\Omega)}^{2} d s\right. \\
& +\|q\|_{L^{2}(\Omega)}^{2}+\|p\|_{H^{1}(\Omega)}^{2}+\int_{0}^{t}\left[\left(H^{-}(s)\right)^{2}+\left(H^{+}(s)\right)^{2}\right] d s .
\end{aligned}
$$

By Gronwall's Lemma, we deduce that

$$
\begin{aligned}
\left\|\partial_{t} \tilde{u}(\cdot, t)\right\|_{L^{2}(\Omega)}^{2} & +\|\tilde{u}(\cdot, t)\|_{H^{1}(\Omega)}^{2}+\int_{0}^{t}\left[\left(\partial_{t} \tilde{u}\left(a_{-}, s\right)\right)^{2}+\left(\partial_{t} \tilde{u}\left(a_{+}, s\right)\right)^{2}\right] d s \\
\leq & e^{T}\left(\|F\|_{L^{2}((0, T) \times \Omega)}^{2}+\|q\|_{L^{2}(\Omega)}^{2}+\|p\|_{H^{1}(\Omega)}^{2}+\sum_{ \pm}\left\|H^{ \pm}\right\|_{L^{2}(0, T)}^{2}\right),
\end{aligned}
$$

which gives

$$
\begin{aligned}
\max _{|\alpha| \leq 1}\left\|\partial^{\alpha} \tilde{u}\right\|_{L^{\infty}\left(0, T ; L^{2}(\Omega)\right)}^{2}+\left\|\partial_{t} \tilde{u}\left(a_{-}, \cdot\right)\right\|_{L^{2}(0, T)}^{2}+\left\|\partial_{t} \tilde{u}\left(a_{+}, \cdot\right)\right\|_{L^{2}(0, T)}^{2} \\
\quad \leq e^{T}\left(\|F\|_{L^{2}((0, T) \times \Omega)}^{2}+\|q\|_{L^{2}(\Omega)}^{2}+\|p\|_{H^{1}(\Omega)}^{2}+\sum_{ \pm}\left\|H^{ \pm}\right\|_{L^{2}(0, T)}^{2}\right) .
\end{aligned}
$$

Differentiating in time in (3.5), we now apply (3.8) to $\partial_{t} \tilde{u}$, and obtain:

$$
\begin{aligned}
& \max _{|\alpha| \leq 1}\left\|\partial^{\alpha} \partial_{t} \tilde{u}\right\|_{L^{\infty}\left(0, T ; L^{2}(\Omega)\right)}^{2}+\left\|\partial_{t}^{2} \tilde{u}\left(a_{-}, \cdot\right)\right\|_{L^{2}(0, T)}^{2}+\left\|\partial_{t}^{2} \tilde{u}\left(a_{+}, \cdot\right)\right\|_{L^{2}(0, T)}^{2} \\
\leq & e^{T}\left(\left\|\partial_{t} F\right\|_{L^{2}((0, T) \times \Omega)}^{2}+\left\|\partial_{t}^{2} \tilde{u}(\cdot, 0)\right\|_{L^{2}(\Omega)}^{2}+\|q\|_{H^{1}(\Omega)}^{2}+\sum_{ \pm}\left\|\partial_{t} H^{ \pm}\right\|_{L^{2}(0, T)}^{2}\right) .
\end{aligned}
$$

We must estimate $\partial_{t}^{2} \tilde{u}(\cdot, 0)$ in the right-hand side of (3.9). We multiply (3.5) by $\partial_{t}^{2} \tilde{u}$, integrate in space using the boundary conditions, and evaluate at time 0 :

$$
\begin{aligned}
\left\|\partial_{t}^{2} \tilde{u}(\cdot, 0)\right\|_{L^{2}(\Omega)}^{2}+ & \left(\partial_{x} p, \partial_{x} \partial_{t}^{2} \tilde{u}(\cdot, 0)\right)+\left(p, \partial_{t}^{2} \tilde{u}(\cdot, 0)\right) \\
& +q\left(a_{-}\right) \partial_{t}^{2} \tilde{u}\left(a_{-}, 0\right)+q\left(a_{+}\right) \partial_{t}^{2} \tilde{u}\left(a_{+}, 0\right) \\
= & H^{-}(0) \partial_{t}^{2} \tilde{u}\left(a_{-}, 0\right)+H^{+}(0) \partial_{t}^{2} \tilde{u}\left(a_{+}, 0\right)+\left(F(\cdot, 0), \partial_{t}^{2} \tilde{u}(\cdot, 0)\right) .
\end{aligned}
$$

We integrate by parts in the second term, and rewrite the equality as

$$
\begin{aligned}
\left\|\partial_{t}^{2} \tilde{u}(\cdot, 0)\right\|_{L^{2}(\Omega)}^{2}-\left(\partial_{x}^{2} \tilde{u}(\cdot, 0), \partial_{t}^{2} \tilde{u}(\cdot, 0)\right)+\left(p, \partial_{t}^{2} \tilde{u}(\cdot, 0)\right) \\
=\left(H^{-}(0)-q\left(a_{-}\right)+\partial_{x} p\left(a_{-}\right)\right) \partial_{t}^{2} \tilde{u}\left(a_{-}, 0\right) \\
\quad+\left(H^{+}(0)-q\left(a_{+}\right)-\partial_{x} p\left(a_{+}\right)\right) \partial_{t}^{2} \tilde{u}\left(a_{+}, 0\right)+\left(F(\cdot, 0), \partial_{t}^{2} \tilde{u}(\cdot, 0)\right) .
\end{aligned}
$$

The boundary terms on the right-hand side vanish by the compatibility conditions, and we get

$$
\left\|\partial_{t}^{2} \tilde{u}(\cdot, 0)\right\|_{L^{2}(\Omega)}^{2}=\left(\partial_{x}^{2} p-p+F(\cdot, 0), \partial_{t}^{2} \tilde{u}(\cdot, 0)\right) .
$$

Using the Cauchy-Schwarz Lemma, we obtain

$$
\left\|\partial_{t}^{2} \tilde{u}(\cdot, 0)\right\|_{L^{2}(\Omega)} \leq\left\|\partial_{x}^{2} p-p+F(\cdot, 0)\right\|_{L^{2}(\Omega)} .
$$


We replace the term $\left\|\partial_{t}^{2} \tilde{u}(\cdot, 0)\right\|_{L^{2}(\Omega)}$ in (3.8), and we deduce the second a priori estimate:

$$
\begin{aligned}
& \text { (3.10) } \max _{|\alpha| \leq 1}\left\|\partial^{\alpha} \partial_{t} \tilde{u}\right\|_{L^{\infty}\left(0, T ; L^{2}(\Omega)\right)}^{2}+\left\|\partial_{t}^{2} \tilde{u}\left(a_{-}, \cdot\right)\right\|_{L^{2}(0, T)}^{2}+\left\|\partial_{t}^{2} \tilde{u}\left(a_{+}, \cdot\right)\right\|_{L^{2}(0, T)}^{2} \\
& \leq 3 e^{T}\left(\left\|\partial_{t} F\right\|_{L^{2}((0, T) \times \Omega)}^{2}+\|F(\cdot, 0)\|_{L^{2}(\Omega)}^{2}+\|p\|_{H^{2}(\Omega)}^{2}+\|q\|_{H^{1}(\Omega)}^{2}+\sum_{ \pm}\left\|\partial_{t} H^{ \pm}\right\|_{L^{2}(0, T)}^{2}\right) .
\end{aligned}
$$

We still need to estimate the mixed derivatives $\partial_{x x} \tilde{u}$ in the interior and $\partial_{x t} \tilde{u}$ on the boundaries. We use the equation, which gives in the interior

$$
\left\|\partial_{x x} \tilde{u}\right\|_{L^{\infty}\left(0, T ; L^{2}(\Omega)\right)} \leq\left\|\partial_{t t} \tilde{u}\right\|_{L^{\infty}\left(0, T ; L^{2}(\Omega)\right)}+\|\tilde{u}\|_{L^{\infty}\left(0, T ; L^{2}(\Omega)\right)}+\|F\|_{L^{\infty}\left(0, T ; L^{2}(\Omega)\right)} .
$$

We now introduce the inequality

$$
\|F\|_{L^{\infty}\left(0, T ; L^{2}(\Omega)\right)}^{2} \leq 2\left(\|F(\cdot, 0)\|_{L^{2}(\Omega)}^{2}+T\left\|\partial_{t} F\right\|_{L^{2}\left(0, T ; L^{2}(\Omega)\right)}^{2}\right),
$$

and by (3.10) and (3.8), we get, using that $e^{T} \geq 1$ and $e^{T} \geq T$,

$$
\begin{aligned}
\left\|\partial_{x x} \tilde{u}\right\|_{L^{\infty}\left(0, T ; L^{2}(\Omega)\right)}^{2} \leq 15 e^{T}\left(\|F\|_{H^{1}\left(0, T ; L^{2}(\Omega)\right)}^{2}+\|F(\cdot, 0)\|_{L^{2}(\Omega)}^{2}\right. & \\
& \left.+\|p\|_{H^{2}(\Omega)}^{2}+\|q\|_{H^{1}(\Omega)}^{2}+\sum_{ \pm}\left\|H^{ \pm}\right\|_{H^{1}(0, T)}^{2}\right) .
\end{aligned}
$$

As for the boundary term, we get, for instance, on the left boundary

$$
\left\|\partial_{t x} \tilde{u}\left(a_{-}, \cdot\right)\right\|_{L^{2}(0, T)} \leq\left\|\partial_{t t} \tilde{u}\left(a_{-}, \cdot\right)\right\|_{L^{2}(0, T)}+\left\|\partial_{t} H^{-}\right\|_{L^{2}(0, T)} .
$$

Squaring the inequality, and adding the term coming from the right boundary leads to

$$
\sum_{ \pm}\left\|\partial_{t x} \tilde{u}\left(a_{ \pm}, \cdot\right)\right\|_{L^{2}(0, T)}^{2} \leq 2 \sum_{ \pm}\left(\left\|\partial_{t t} \tilde{u}\left(a_{ \pm}, \cdot\right)\right\|_{L^{2}(0, T)}^{2}+\left\|\partial_{t} H^{ \pm}\right\|_{L^{2}(0, T)}^{2}\right),
$$

which provides the last estimate announced in the proposition. The well-posedness is then derived in a standard way by the Galerkin method.

The solution $\bar{u}$ of the nonlinear subdomain problem is now defined through an iterative scheme. The initial guess is $\bar{u}_{0}=p$. At step $k, \bar{u}_{k}$ being known, we define (3.11)

$$
\mathcal{F}(w)=w+f\left(w, \partial_{t} w, \partial_{x} w\right), \mathcal{G}^{ \pm}(w)=g^{ \pm}\left(w\left(a_{ \pm}, \cdot\right)\right), \mathcal{H}^{ \pm}(w)=h^{ \pm}-\mathcal{G}^{ \pm}(w) .
$$

$\bar{u}_{k+1}$ is the solution of the linear initial boundary value problem (3.5) with data $f_{k}=\mathcal{F}\left(\bar{u}_{k}\right), g_{k}^{ \pm}=\mathcal{G}^{ \pm}\left(\bar{u}_{k}\right), h_{k}^{ \pm}=\mathcal{H}\left(\bar{u}_{k}\right)$, and initial data $p$ and $q$. The proof of convergence for the sequence $\bar{u}_{k}$ is written in detail in [12. The uniqueness follows from the result:

Lemma 3.3. There exists a real positive increasing function $\theta$ such that, for any time $T$, for any $v$ in $V_{2}(\Omega, T), \partial^{\alpha} \mathcal{F}(v)$ is in $V_{1}(\Omega, T), \mathcal{G}^{ \pm}(v)$ and $\mathcal{H}(v)$ are in $L^{\infty}(0, T)$. Moreover, for $v_{1}, v_{2}$ in $V_{2}(\Omega, T)$, we have

$$
\begin{gathered}
\left\|\mathcal{H}^{ \pm}\left(v_{1}\right)-\mathcal{H}^{ \pm}\left(v_{2}\right)\right\|_{L^{\infty}(0, T)} \leq \theta\left(\left\|v_{1}\right\|_{V_{2}(\Omega, T)}+\left\|v_{2}\right\|_{V_{2}(\Omega, T)}\right)\left\|v_{1}-v_{2}\right\|_{V_{1}(\Omega, T)}, \\
\left\|\mathcal{F}\left(v_{1}\right)-\mathcal{F}\left(v_{2}\right)\right\|_{V_{1}(\Omega, T)} \leq \theta\left(\left\|v_{1}\right\|_{V_{2}(\Omega, T)}+\left\|v_{2}\right\|_{V_{2}(\Omega, T)}\right)\left\|v_{1}-v_{2}\right\|_{V_{2}(\Omega, T)} .
\end{gathered}
$$

As a consequence, we have the well-posedness of problem (2.4). 
Corollary 3.4. Let $p$ be in $H_{0}^{2}(\Omega)$ and $q$ be in $H_{0}^{1}(\Omega)$. There exists a time $T_{0}^{*}>0$ such that for any $T \leq T_{0}^{*}$, (2.4) has a unique solution $\bar{u}$ in $V_{2}(\Omega, T)$, with $\partial^{\alpha} \bar{u}(a, \cdot)$ and $\partial^{\alpha} \bar{u}(b, \cdot)$ in $H^{1}(0, T)$ for $|\alpha|=1$. Furthermore, there exists a positive real number $C^{*}$ depending only on the size of $\Omega$ such that

$$
\begin{aligned}
& \|\bar{u}\|_{V_{2}(\Omega, T)}^{2}+\sum_{|\alpha|=1}\left\|\partial^{\alpha} \bar{u}(a, \cdot)\right\|_{H^{1}(0, T)}^{2} \sum_{|\alpha|=1}\left\|\partial^{\alpha} \bar{u}(b, \cdot)\right\|_{H^{1}(0, T)}^{2} \\
& \quad \leq C^{*}\left(\|p\|_{H^{2}(\Omega)}^{2}+\|q\|_{H^{1}(\Omega)}^{2}\right) .
\end{aligned}
$$

\section{Convergence of the Algorithm}

We now study the convergence of the Schwarz waveform relaxation algorithm (2.5). In order to define the algorithm, we need a regularity result:

Proposition 4.1. For any $\epsilon, 0<\epsilon<1$,

$$
V_{2}(\Omega, T) \subset \mathcal{C}^{0}\left(0, T ; H^{2-\epsilon}(\Omega)\right) \cap \mathcal{C}^{1}\left(0, T ; H^{1-\epsilon}(\Omega)\right) .
$$

Proof. By using extension operators in time and space, it suffices to prove the result in $\mathbb{R} \times \mathbb{R}$. We make use of the Littlewood-Paley theory (see for example [2]). In particular, there exists $\varphi$ and $\chi$ two tempered distributions on $\mathbb{R}$, with $\varphi$ supported in $(-8 / 3,-3 / 4) \cup(3 / 4,8 / 3), \chi$ supported in $(-4 / 3,4 / 3)$, and

$$
\chi(\xi)+\sum_{q \geq 0} \varphi\left(2^{-q} \xi\right)=1, \forall \xi \in \mathbb{R} .
$$

We define the dyadic projectors $\Delta_{q}$ by their action on a function $\mathrm{u}$,

$$
\Delta_{-1} u=\chi(D) u, \Delta_{q} u=\varphi\left(2^{-q} D\right) u \text { for } q \geq 0,
$$

where $D=-i \partial$. These operators give an equivalent norm in $H^{s}(\mathbb{R})$,

$$
|u|_{s}=\left(\sum_{q \geq-1} 2^{2 q s}\left\|\Delta_{q} u\right\|^{2}\right)^{\frac{1}{2}} .
$$

They can also be used to define the Zygmund spaces

$$
\mathcal{C}_{*}^{r}=\left\{u \in \mathcal{S}^{\prime},\|u\|_{r}=\sup _{q \geq-1} 2^{q r}\left\|\Delta_{q} u\right\|^{2}<+\infty\right\} .
$$

$\mathcal{C}_{*}^{r}$ coincides with the usual Hölder space when $r$ is not an integer. For any positive $r$, we know that $W^{r, \infty}$, the space of functions in $L^{\infty}$ with derivatives of order up to $r$ in $L^{\infty}$, is included in $\mathcal{C}_{*}^{r}$. Therefore we have

$$
V_{2}(\mathbb{R}, \mathbb{R}) \subset \mathcal{C}_{*}^{0}\left(\mathbb{R}, H^{2}(\mathbb{R})\right) \cap \mathcal{C}_{*}^{1}\left(\mathbb{R}, H^{1}(\mathbb{R})\right) \cap \mathcal{C}_{*}^{2}\left(\mathbb{R}, L^{2}(\mathbb{R})\right) .
$$

We need an interpolation lemma.

Lemma 4.2. For any positive $\alpha, \beta, a, b$, for any $\theta, 0 \leq \theta \leq 1$,

$$
\mathcal{C}_{*}^{\alpha}\left(\mathbb{R}, H^{a}(\mathbb{R})\right) \cap \mathcal{C}_{*}^{\beta}\left(\mathbb{R}, H^{b}(\mathbb{R})\right) \subset \mathcal{C}_{*}^{\theta \alpha+(1-\theta) \beta}\left(\mathbb{R}, H^{\theta a+(1-\theta) b}(\mathbb{R})\right) .
$$

Applying the lemma with successively $(\alpha, \beta, a, b)=(0,1,2,1)$ and $(\alpha, \beta, a, b)=$ $(1,2,1,0)$, we find for any $\theta, \theta^{\prime}$ in $(0,1)$,

$$
V_{2}(\mathbb{R}, \mathbb{R}) \subset \mathcal{C}_{*}^{1-\theta}\left(\mathbb{R}, H^{1+\theta}(\mathbb{R})\right) \cap \mathcal{C}_{*}^{2-\theta^{\prime}}\left(\mathbb{R}, H^{\theta^{\prime}}(\mathbb{R})\right)
$$

Since for any $\epsilon>0$ we have $\mathcal{C}_{*}^{\epsilon} \subset \mathcal{C}^{0}$ and $\mathcal{C}_{*}^{1+\epsilon} \subset \mathcal{C}^{1}$, this concludes the proof of Proposition 4.1 . 
Proof of Lemma 4.2. It relies on the convexity of the exponential function

$$
\|u\|_{\mathcal{C}_{*}^{\theta \alpha+(1-\theta) \beta}\left(\mathbb{R}, H^{\theta a+(1-\theta) b(\mathbb{R}))}\right.}^{2}=\sup _{j \geq-1} 2^{2 j(\theta \alpha+(1-\theta) \beta)} \sum_{k \geq-1} 2^{2 k(\theta a+(1-\theta) b)}\left\|\Delta_{j}^{t} \Delta_{k}^{x} u\right\|^{2}
$$

where $\Delta_{j}^{t}$ (resp. $\Delta_{j}^{x}$ ) is the Littlewood-Paley operator acting in the time (resp. space) variable.

$$
\begin{aligned}
\sum_{k \geq-1} 2^{2 k(\theta a+(1-\theta) b)}\left\|\Delta_{j}^{t} \Delta_{k}^{x} u\right\|^{2} & =\sum_{k \geq-1}\left(\left(2^{2 k a}\left\|\Delta_{j}^{t} \Delta_{k}^{x} u\right\|^{2}\right)^{\theta}\left(2^{2 k b}\left\|\Delta_{j}^{t} \Delta_{k}^{x} u\right\|^{2}\right)^{(1-\theta)}\right) \\
& \leq\left(\sum_{k \geq-1} 2^{2 k a}\left\|\Delta_{j}^{t} \Delta_{k}^{x} u\right\|^{2}\right)^{\theta}\left(\sum_{k \geq-1} 2^{2 k b}\left\|\Delta_{j}^{t} \Delta_{k}^{x} u\right\|^{2}\right)^{1-\theta} .
\end{aligned}
$$

Therefore we have

$$
\begin{aligned}
& \|u\|_{\mathcal{C}_{*}^{\theta \alpha+(1-\theta) \beta}\left(\mathbb{R}, H^{\theta a+(1-\theta) b}(\mathbb{R})\right)}^{2} \\
& \quad \leq\left(\sup _{j \geq-1} 2^{2 j \alpha} \sum_{k \geq-1} 2^{2 k a}\left\|\Delta_{j}^{t} \Delta_{k}^{x} u\right\|^{2}\right)^{\theta}\left(\sup _{j \geq-1} 2^{2 j \beta} \sum_{k \geq-1} 2^{2 k b}\left\|\Delta_{j}^{t} \Delta_{k}^{x} u\right\|^{2}\right)^{1-\theta}
\end{aligned}
$$

which gives

$$
\|u\|_{\mathcal{C}_{*}^{\theta \alpha+(1-\theta) \beta}\left(\mathbb{R}, H^{\theta a+(1-\theta) b(\mathbb{R}))}\right.}^{2} \leq\left(\|u\|_{\mathcal{C}_{*}^{\alpha}\left(\mathbb{R}, H^{a}(\mathbb{R})\right)}^{2}\right)^{\theta}\left(\|u\|_{\mathcal{C}_{*}^{\beta}\left(\mathbb{R}, H^{b}(\mathbb{R})\right)}^{2}\right)^{1-\theta} .
$$

Theorem 4.3. Let $p$ be in $H_{0}^{2}(\Omega)$ and $q$ be in $H_{0}^{1}(\Omega)$. There exists a time $T_{1} \leq T_{0}^{*}$ such that for any $T \leq T_{1}$, for any initial guess $h_{i}^{ \pm}$be in $H^{1}(0, T)$ with the compatibility conditions $h_{i}^{+}(0)=q\left(a_{i+1}\right)+p^{\prime}\left(a_{i+1}\right)+g^{+}\left(p\left(a_{i+1}\right)\right)$ and $h_{i}^{-}(0)=q\left(a_{i}\right)-$ $p^{\prime}\left(a_{i}\right)+g^{-}\left(p\left(a_{i}\right)\right)$, the algorithm (2.5) is defined and converges in $\bigcup_{i} V_{2}\left(\Omega_{i}, T\right)$ to the solution $\bar{u}$ of (2.4).

Proof. We first prove that the algorithm is well defined: with the assumptions on $h_{i}^{ \pm}$in the theorem, we know by Theorem 3.1 that (2.5) defines in each $\Omega_{i}$ a $u_{i}^{1}$ in $V_{2}\left(\Omega_{i}, T\right)$, with $\partial_{t} u_{i}^{1}\left(a_{i}, \cdot\right), \partial_{t} u_{i}^{1}\left(a_{i+1}, \cdot\right), \partial_{x} u_{i}^{1}\left(a_{i}, \cdot\right)$ and $\partial_{x} u_{i}^{1}\left(a_{i+1}, \cdot\right)$ in $H^{1}(0, T)$ for $T \leq T_{i}^{1}$. Furthermore, by Lemma 3.3. $\mathcal{B}^{-}\left(g^{-}\right) u_{i-1}^{1}\left(a_{i}, \cdot\right)$ and $\mathcal{B}^{+}\left(g^{+}\right) u_{i+1}^{1}\left(a_{i+1}, \cdot\right)$ are in $H^{1}(0, T)$. As for the compatibility conditions, we have

$$
\mathcal{B}^{-}\left(g^{-}\right) u_{i-1}^{1}\left(a_{i}, 0\right)=\lim _{t \rightarrow 0}\left(\partial_{t} u_{i-1}^{1}\left(a_{i}, t\right)-\partial_{x} u_{i-1}^{1}\left(a_{i}, t\right)+g^{-}\left(u_{i-1}^{1}\left(a_{i}, t\right)\right)\right),
$$

and by Proposition 4.1, we can pass to the limit and get

$$
\mathcal{B}^{-}\left(g^{-}\right) u_{i-1}^{1}\left(a_{i}, 0\right)=q\left(a_{i}\right)-p^{\prime}\left(a_{i}\right)+g^{-}\left(p\left(a_{i}\right)\right) \text {. }
$$

This, together with the same regularity result on $a_{i+1}$, permits the recursion.

We define for $T \leq \min \left(T_{0}^{*}, \min _{i}\left(T_{i}^{* k}\right)\right.$ ), for $k \geq 1$, the quantities (with $u_{i}=\bar{u} / \Omega_{i}$ ) for $1 \leq i, j \leq I, j=i$ or $j=i-1$,

$$
\begin{aligned}
e_{i}^{k} & =u_{i}^{k}-u_{i}, \\
f_{i}^{k} & =\mathcal{F}\left(u_{i}^{k}\right)-\mathcal{F}\left(u_{i}\right), \\
h_{i, j}^{k,-} & =g^{-}\left(u_{j}^{k}\left(a_{i}\right)\right)-g^{-}\left(u_{j}\left(a_{i}\right)\right), \\
h_{i, j}^{k,+} & =g^{+}\left(u_{j}^{k}\left(a_{i+1}\right)\right)-g^{+}\left(u_{j}\left(a_{i+1}\right)\right) .
\end{aligned}
$$


The operator $\mathcal{F}$ is defined in (3.11). The error $e_{i}^{k}$ in $\Omega_{i}$ at iteration $k$ is a solution of

$$
\begin{aligned}
& \left(\partial_{t}^{2}-\partial_{x}^{2}\right) e_{i}^{k}+e_{i}^{k}=f_{i}^{k} \text { in } \Omega_{i} \times(0, T), \\
& \left(\partial_{t}-\partial_{x}\right) e_{i}^{k}+h_{i, i}^{k,-}=\left(\partial_{t}-\partial_{x}\right) e_{i-1}^{k-1}+h_{i, i-1}^{k-1,-} \text { on }\left\{a_{i}\right\} \times(0, T), \\
& \left(\partial_{t}+\partial_{x}\right) e_{i}^{k}+h_{i, i}^{k,+}=\left(\partial_{t}+\partial_{x}\right) e_{i+1}^{k-1}+h_{i, i+1}^{k-1,+} \text { on }\left\{a_{i+1}\right\} \times(0, T),
\end{aligned}
$$

with vanishing initial values and $e_{0}^{k} \equiv 0, e_{I+2}^{k} \equiv 0, h_{1,0}^{k,-} \equiv 0, h_{I I+1}^{k,+}=0$. In order to get a new energy estimate in $\Omega_{i}$, we multiply (4.2) by $\partial_{t} e_{i}^{k}$ and integrate by parts:

$$
\frac{d}{d t} E_{\Omega_{i}}\left(e_{i}^{k}\right)-\left[\partial_{t} e_{i}^{k} \partial_{x} e_{i}^{k}\left(a_{i+1}, \cdot\right)-\partial_{t} e_{i}^{k} \partial_{x} e_{i}^{k}\left(a_{i}, \cdot\right)\right]=\left(f_{i}^{k}, \partial_{t} e_{i}^{k}\right)
$$

with $E_{\Omega}(u)=\frac{1}{2}\left(\left\|\partial_{t} u\right\|_{L^{2}(\Omega)}^{2}+\left\|\partial_{x} u\right\|_{L^{2}(\Omega)}^{2}+\|u\|_{L^{2}(\Omega)}^{2}\right)$. We rewrite the boundary terms using the boundary operators:

$$
\begin{aligned}
\partial_{t} e_{i}^{k} \partial_{x} e_{i}^{k}\left(a_{i+1}, \cdot\right)= & \frac{1}{4}\left(\left(\partial_{t}+\partial_{x}\right) e_{i}^{k}\left(a_{i+1}, \cdot\right)+h_{i, i}^{k,+}\right)^{2} \\
& -\frac{1}{4}\left(\left(\partial_{t}-\partial_{x}\right) e_{i}^{k}\left(a_{i+1}, \cdot\right)+h_{i+1, i}^{k,-}\right)^{2}+R_{i, i+1}^{k}, \\
-\partial_{t} e_{i}^{k} \partial_{x} e_{i}^{k}\left(a_{i}, \cdot\right)= & \frac{1}{4}\left(\left(\partial_{t}-\partial_{x}\right) e_{i}^{k}\left(a_{i}, \cdot\right)+h_{i, i}^{k,-}\right)^{2} \\
& -\frac{1}{4}\left(\left(\partial_{t}+\partial_{x}\right) e_{i}^{k}\left(a_{i}, \cdot\right)+h_{i-1, i}^{k,+}\right)^{2}+R_{i, i-1}^{k}
\end{aligned}
$$

The remainders $R_{i, i+1}^{k}$ and $R_{i, i-1}^{k}$ will be evaluated later. We insert (4.6) into (4.5), and obtain

$$
\begin{aligned}
\frac{d}{d t} E_{\Omega_{i}}\left(e_{i}^{k}\right)+ & \frac{1}{4}\left(\left(\partial_{t}-\partial_{x}\right) e_{i}^{k}\left(a_{i+1}, \cdot\right)+h_{i+1, i}^{k,-}\right)^{2}+\frac{1}{4}\left(\left(\partial_{t}+\partial_{x}\right) e_{i}^{k}\left(a_{i}, \cdot\right)+h_{i-1, i}^{k,+}\right)^{2} \\
= & \frac{1}{4}\left(\left(\partial_{t}+\partial_{x}\right) e_{i}^{k}\left(a_{i+1}, \cdot\right)+h_{i, i}^{k,+}\right)^{2}+\frac{1}{4}\left(\left(\partial_{t}-\partial_{x}\right) e_{i}^{k}\left(a_{i}, \cdot\right)+h_{i, i}^{k,-}\right)^{2} \\
& +R_{i, i+1}^{k}+R_{i, i-1}^{k}+\left(f_{i}^{k}, \partial_{t} e_{i}^{k}\right) .
\end{aligned}
$$

Using the transmission conditions (4.3), (4.4), we get

$$
\begin{aligned}
\frac{d}{d t} E_{\Omega_{i}}\left(e_{i}^{k}\right)+ & \frac{1}{4}\left(\left(\partial_{t}-\partial_{x}\right) e_{i}^{k}\left(a_{i+1}, \cdot\right)+h_{i+1, i}^{k,-}\right)^{2} \\
& +\frac{1}{4}\left(\left(\partial_{t}+\partial_{x}\right) e_{i}^{k}\left(a_{i}, \cdot\right)+h_{i-1, i}^{k,+}\right)^{2} \\
= & \frac{1}{4}\left(\left(\partial_{t}+\partial_{x}\right) e_{i+1}^{k-1}\left(a_{i+1}, \cdot\right)+h_{i, i+1}^{k-1,+}\right)^{2} \\
& +\frac{1}{4}\left(\left(\partial_{t}-\partial_{x}\right) e_{i-1}^{k-1}\left(a_{i}, \cdot\right)+h_{i, i-1}^{k-1,-}\right)^{2} \\
& +R_{i, i+1}^{k}+R_{i, i-1}^{k}+\left(f_{i}^{k}, \partial_{t} e_{i}^{k}\right) .
\end{aligned}
$$

We sum (4.7) on the indexes $i, 1 \leq i \leq I$ and integrate in time. We translate the 
domain indexes in the right-hand side. Defining

$$
E_{\partial \Omega_{i}}\left(e_{i}^{k}\right)=\frac{1}{4}\left[\left(\left(\partial_{t}-\partial_{x}\right) e_{i}^{k}\left(a_{i+1}, \cdot\right)+h_{i+1, i}^{k,-}\right)^{2}+\left(\left(\partial_{t}+\partial_{x}\right) e_{i}^{k}\left(a_{i}, \cdot\right)+h_{i-1, i}^{k,+}\right)^{2}\right],
$$

we get, since the initial data vanish,

$$
\begin{aligned}
\sum_{i=1}^{I} E_{\Omega_{i}}\left(e_{i}^{k}\right)(t) & +\int_{0}^{t} \sum_{i=1}^{I} E_{\partial \Omega_{i}}\left(e_{i}^{k}\right)(s) d s \leq \int_{0}^{t} \sum_{i=1}^{I} E_{\partial \Omega_{i}}\left(e_{i}^{k-1}\right)(s) d s \\
& +\int_{0}^{t} \sum_{i=1}^{I}\left(R_{i, i+1}^{k}+R_{i, i-1}^{k}\right)(s) d s+\int_{0}^{t} \sum_{i=1}^{I}\left(f_{i}^{k}, \partial_{t} e_{i}^{k}\right)(s) d s .
\end{aligned}
$$

Differentiating the equation and the transmission conditions in time yields the bound on $\partial_{t} e_{i}^{k}$ :

$$
\begin{aligned}
\sum_{i=1}^{I} E_{\Omega_{i}}\left(\partial_{t} e_{i}^{k}\right)(t) & +\int_{0}^{t} \sum_{i=1}^{I} E_{\partial \Omega_{i}}\left(\partial_{t} e_{i}^{k}\right)(s) d s \leq \int_{0}^{t} \sum_{i=1}^{I} E_{\partial \Omega_{i}}\left(\partial_{t} e_{i}^{k-1}\right)(s) d s \\
& +\int_{0}^{t} \sum_{i=1}^{I}\left(\tilde{R}_{i, i+1}^{k}+\tilde{R}_{i, i-1}^{k}\right)(s) d s+\int_{0}^{t} \sum_{i=1}^{I}\left(\partial_{t} f_{i}^{k+1}, \partial_{t t} e_{i}^{k}\right)(s) d s .
\end{aligned}
$$

We now estimate the remainders. We start with $R_{i, i+1}^{k}$ (ignoring the superscript $k)$ :

$R_{i, i+1}=\frac{1}{4}\left[-h_{i, i}^{+}\left(2\left(\partial_{t} e_{i}+\partial_{x} e_{i}\right)\left(a_{i+1}, \cdot\right)+h_{i, i}^{+}\right)+h_{i+1, i}^{-}\left(2\left(\partial_{t} e_{i}-\partial_{x} e_{i}\right)\left(a_{i+1}, \cdot\right)+h_{i+1, i}^{-}\right)\right]$, and we get a bound on the integral of $R_{i, i+1}$ :

$$
\begin{aligned}
\int_{0}^{t} R_{i, i+1}(s) \leq & \frac{3}{4}\left(\left\|h_{i, i}^{+}\right\|_{H^{\frac{1}{2}}(0, t)}^{2}+\left\|h_{i+1, i}^{-}\right\|_{H^{\frac{1}{2}}(0, t)}^{2}\right) \\
& +\frac{1}{2}\left(\left\|\partial_{t} e_{i}\left(a_{i+1}, \cdot\right)\right\|_{H^{-\frac{1}{2}}(0, t)}^{2}+\left\|\partial_{x} e_{i}\left(a_{i+1}, \cdot\right)\right\|_{H^{-\frac{1}{2}}(0, t)}^{2}\right) .
\end{aligned}
$$

We can treat $R_{i, i-1}, \tilde{R}_{i, i-1}$, and $\tilde{R}_{i, i-1}$ the same way and obtain

$$
\begin{aligned}
\int_{0}^{t} R_{i, i-1}(s) \leq & \frac{3}{4}\left(\left\|h_{i, i}^{-}\right\|_{H^{\frac{1}{2}}(0, t)}^{2}+\left\|h_{i-1, i}^{+}\right\|_{H^{\frac{1}{2}}(0, t)}^{2}\right) \\
& +\frac{1}{2}\left(\left\|\partial_{t} e_{i}\left(a_{i}, \cdot\right)\right\|_{H^{-\frac{1}{2}}(0, t)}^{2}+\left\|\partial_{x} e_{i}\left(a_{i}, \cdot\right)\right\|_{H^{-\frac{1}{2}}(0, t)}^{2}\right), \\
\int_{0}^{t} \tilde{R}_{i, i+1}(s) \leq & \frac{3}{4}\left(\left\|\partial_{t} h_{i, i}^{+}\right\|_{H^{\frac{1}{2}}(0, t)}^{2}+\left\|\partial_{t} h_{i+1, i}^{-}\right\|_{H^{\frac{1}{2}}(0, t)}^{2}\right) \\
& +\frac{1}{2}\left(\left\|\partial_{t}^{2} e_{i}\left(a_{i+1}, \cdot\right)\right\|_{H^{-\frac{1}{2}}(0, t)}^{2}+\left\|\partial_{x t} e_{i}\left(a_{i+1}, \cdot\right)\right\|_{H^{-\frac{1}{2}}(0, t)}^{2}\right), \\
\int_{0}^{t} \tilde{R}_{i, i-1}(s) \leq & \frac{3}{4}\left(\left\|\partial_{t} h_{i, i}^{-}\right\|_{H^{\frac{1}{2}}(0, t)}^{2}+\left\|\partial_{t} h_{i-1, i}^{+}\right\|_{H^{\frac{1}{2}}(0, t)}^{2}\right) \\
& +\frac{1}{2}\left(\left\|\partial_{t}^{2} e_{i}\left(a_{i}, \cdot\right)\right\|_{H^{-\frac{1}{2}}(0, t)}^{2}+\left\|\partial_{x t} e_{i}\left(a_{i}, \cdot\right)\right\|_{H^{-\frac{1}{2}}(0, t)}^{2}\right) .
\end{aligned}
$$


At point $a_{i}$, for instance, by the Trace Theorem, there is a constant $C_{3}$ independent of $T$, such that for any $\alpha$ with $|\alpha|=1$, we have

$$
\begin{gathered}
\left\|\partial^{\alpha} e_{i}\left(a_{i}, \cdot\right)\right\|_{H^{-\frac{1}{2}}(0, t)} \leq\left\|\partial^{\alpha} e_{i}\left(a_{i}, \cdot\right)\right\|_{L^{2}(0, t)} \leq C_{3}\left\|\partial^{\alpha} e_{i}\right\|_{H^{1}\left(\Omega_{i} \times(0, t)\right)}, \\
\left\|\partial^{\alpha} \partial_{t} e_{i}\left(a_{i}, \cdot\right)\right\|_{H^{-\frac{1}{2}}(0, t)} \leq\left\|\partial^{\alpha} e_{i}\left(a_{i}, \cdot\right)\right\|_{H^{\frac{1}{2}}(0, t)} \leq C_{3}\left\|\partial^{\alpha} e_{i}\right\|_{H^{1}\left(\Omega_{i} \times(0, t)\right)},
\end{gathered}
$$

which gives our first bounds on the remainders:

$$
\begin{gathered}
\int_{0}^{t} R_{i, i+1}(s) d s \leq \frac{1}{2}\left(\left\|h_{i, i}^{+}\right\|_{H^{\frac{1}{2}}(0, t)}^{2}+\left\|h_{i+1, i}^{-}\right\|_{H^{\frac{1}{2}}(0, t)}^{2}\right)+\frac{C_{3}^{2}}{2}\left\|e_{i}\right\|_{H^{2}\left(\Omega_{i} \times(0, t)\right)}^{2}, \\
\int_{0}^{t} \tilde{R}_{i, i+1}(s) d s \leq \frac{1}{2}\left(\left\|\partial_{t} h_{i, i}^{+}\right\|_{H^{\frac{1}{2}}(0, t)}^{2}+\left\|\partial_{t} h_{i+1, i}^{-}\right\|_{H^{\frac{1}{2}}(0, t)}^{2}\right)+\frac{C_{3}^{2}}{2}\left\|e_{i}\right\|_{H^{2}\left(\Omega_{i} \times(0, t)\right)}^{2} .
\end{gathered}
$$

We now insert the previous estimates in (4.8) and (4.9). By Cauchy-Schwarz inequality we get

$$
\begin{aligned}
& \sum_{i=1}^{I} E_{\Omega_{i}}\left(e_{i}^{k}\right)(t)+\int_{0}^{t} \sum_{i=1}^{I} E_{\partial \Omega_{i}}\left(e_{i}^{k}\right)(s) d s \\
& \leq \int_{0}^{t} \sum_{i=1}^{I} E_{\partial \Omega_{i}}\left(e_{i}^{k-1}\right)(s) d s+\frac{C_{3}^{2}+1}{2} \sum_{i=1}^{I}\left\|e_{i}^{k}\right\|_{H^{2}\left(\Omega_{i} \times(0, t)\right)}^{2} \\
&+\frac{1}{2} \sum_{i=1}^{I} \int_{0}^{t}\left\|f_{i}^{k}\right\|_{L^{2}\left(\Omega_{i}\right)}^{2}(s) d s \\
&+\frac{3}{4} \sum_{i=1}^{I}\left(\left\|h_{i, i}^{k,+}\right\|_{H^{\frac{1}{2}(0, t)}}^{2}+\left\|h_{i+1, i}^{k,-}\right\|_{H^{\frac{1}{2}}(0, t)}^{2}\right) \\
&+\frac{3}{4} \sum_{i=1}^{I}\left(\left\|h_{i, i}^{k,-}\right\|_{H^{\frac{1}{2}}(0, t)}^{2}+\left\|h_{i-1, i}^{k,+}\right\|_{H^{\frac{1}{2}}(0, t)}^{2}\right) \\
& \sum_{i=1}^{I} E_{\Omega_{i}}\left(\partial_{t} e_{i}^{k}\right)(t)+\int_{0}^{t} \sum_{i=1}^{I} E_{\partial \Omega_{i}}\left(\partial_{t} e_{i}^{k}\right)(s) d s \\
& \leq \int_{0}^{t} \sum_{i=1}^{I} E_{\partial \Omega_{i}}\left(\partial_{t} e_{i}^{k-1}\right)(s) d s+\frac{C_{3}^{2}+1}{2} \sum_{i=1}^{I}\left\|e_{i}^{k}\right\|_{H^{2}\left(\Omega_{i} \times(0, t)\right)}^{2} \\
&+\frac{1}{2} \sum_{i=1}^{I} \int_{0}^{t}\left\|\partial_{t} f_{i}^{k}\right\|_{L^{2}\left(\Omega_{i}\right)}^{2}(s) d s \\
&+\frac{3}{4} \sum_{i=1}^{I}\left(\left\|\partial_{t} h_{i, i}^{k,+}\right\|_{H^{\frac{1}{2}}(0, t)}^{2}+\left\|\partial_{t} h_{i+1, i}^{k,-}\right\|_{H^{\frac{1}{2}}(0, t)}^{2}\right) \\
&+\frac{3}{4} \sum_{i=1}^{I}\left(\left\|\partial_{t} h_{i, i}^{k,-}\right\|_{H^{\frac{1}{2}}(0, t)}^{2}+\left\|\partial_{t} h_{i-1, i}^{k,+}\right\|_{H^{\frac{1}{2}}(0, t)}^{2}\right) .
\end{aligned}
$$


Adding (4.10) and (4.11), we can write

$$
\begin{aligned}
& \sum_{i=1}^{I}\left(E_{\Omega_{i}}\left(e_{i}^{k}\right)+E_{\Omega_{i}}\left(\partial_{t} e_{i}^{k}\right)\right)(t)+\int_{0}^{t} \sum_{i=1}^{I}\left(E_{\partial \Omega_{i}}\left(e_{i}^{k}\right)+E_{\partial \Omega_{i}}\left(\partial_{t} e_{i}^{k}\right)\right)(s) d s \\
& \leq \int_{0}^{t} \sum_{i=1}^{I}\left(E_{\partial \Omega_{i}}\left(e_{i}^{k-1}\right)+E_{\partial \Omega_{i}}\left(\partial_{t} e_{i}^{k-1}\right)\right)(s) d s+\left(C_{3}^{2}+1\right) \sum_{i=1}^{I}\left\|e_{i}^{k}\right\|_{H^{2}\left(\Omega_{i} \times(0, t)\right)}^{2} \\
& +\frac{3}{4} \sum_{i=1}^{I}\left(\left\|h_{i, i}^{k,+}\right\|_{H^{\frac{1}{2}(0, t)}}^{2}+\left\|h_{i+1, i}^{k,+}\right\|_{H^{\frac{1}{2}(0, t)}}^{2}+\left\|h_{i, i}^{k,-}\right\|_{H^{\frac{1}{2}(0, t)}}^{2}+\left\|h_{i-1, i}^{k,+}\right\|_{H^{\frac{1}{2}(0, t)}}^{2}\right) \\
& +\frac{3}{4} \sum_{i=1}^{I}\left(\left\|\partial_{t} h_{i, i}^{k,+}\right\|_{H^{\frac{1}{2}(0, t)}}^{2}+\left\|\partial_{t} h_{i+1, i}^{k,+}\right\|_{H^{\frac{1}{2}}(0, t)}^{2}+\left\|\partial_{t} h_{i, i}^{k,-}\right\|_{H^{\frac{1}{2}(0, t)}}^{2}+\left\|\partial_{t} h_{i-1, i}^{k,+}\right\|_{H^{\frac{1}{2}}(0, t)}^{2}\right) \\
& +\sum_{i=1}^{I} \frac{1}{2}\left(\left\|f_{i}^{k}\right\|_{L^{2}(\Omega \times(0, T))}^{2}+\left\|\partial_{t} f_{i}^{k}\right\|_{L^{2}(\Omega \times(0, T))}^{2}\right) .
\end{aligned}
$$

We now estimate the quantities involving the $h_{i, j}^{k, \pm}$. Refining the results in Lemma 3.3. we have a real positive increasing function $\theta_{2}$, such that

$$
\begin{aligned}
&\left\|h_{i, j}^{k, \pm}\right\|_{H^{\frac{1}{2}}(0, t)}^{2}+\left\|\partial_{t} h_{i, j}^{k, \pm}\right\|_{H^{\frac{1}{2}}(0, t)}^{2} \\
& \leq \theta_{2}^{2}\left(\sum_{\substack{|\alpha| \leq 2 \\
\alpha \neq(0,2)}}\left(\left\|\partial^{\alpha} u_{i}\right\|_{L^{2}(\Omega \times(0, T))}^{2}+\left\|\partial^{\alpha} u_{i}^{k}\right\|_{L^{2}(\Omega \times(0, T))}^{2}\right)\right) \\
& \times \sum_{\substack{|\alpha| \leq 2 \\
\alpha \neq(0,2)}}\left\|\partial^{\alpha} e_{i}^{k}\right\|_{L^{2}(\Omega \times(0, T))}^{2}
\end{aligned}
$$

which gives

$$
\begin{aligned}
& \sum_{i=1}^{I}\left(E_{\Omega_{i}}\left(e_{i}^{k}\right)+E_{\Omega_{i}}\left(\partial_{t} e_{i}^{k}\right)\right)(t)+\int_{0}^{t} \sum_{i=1}^{I}\left(E_{\partial \Omega_{i}}\left(e_{i}^{k}\right)+E_{\partial \Omega_{i}}\left(\partial_{t} e_{i}^{k}\right)\right)(s) d s \\
& \quad \leq \int_{0}^{t} \sum_{i=1}^{I}\left(E_{\partial \Omega_{i}}\left(e_{i}^{k-1}\right)+E_{\partial \Omega_{i}}\left(\partial_{t} e_{i}^{k-1}\right)\right)(s) d s \\
& \quad+\sum_{i=1}^{I} \theta_{3}\left(\sum_{\substack{|\alpha| \leq 2 \\
\alpha \neq(0,2)}}\left(\left\|\partial^{\alpha} u_{i}\right\|_{L^{2}(\Omega \times(0, T))}^{2}+\left\|\partial^{\alpha} u_{i}^{k}\right\|_{L^{2}(\Omega \times(0, T))}^{2}\right)\right)\left\|e_{i}^{k}\right\|_{H^{2}\left(\Omega_{i} \times(0, t)\right)}^{2} \\
& \quad+\sum_{i=1}^{I} \frac{1}{2}\left(\left\|f_{i}^{k}\right\|_{L^{2}(\Omega \times(0, T))}^{2}+\left\|\partial_{t} f_{i}^{k}\right\|_{L^{2}(\Omega \times(0, T))}^{2}\right),
\end{aligned}
$$

with $\theta_{3}=3 \theta_{2}^{2}+C_{3}^{2}+1$. We now evaluate the terms on the right-hand side. We first note that

$$
\left\|e_{i}^{k}\right\|_{H^{2}\left(\Omega_{i} \times(0, t)\right)}^{2} \leq \int_{0}^{t}\left(E_{\Omega_{i}}\left(e_{i}^{k}\right)+E_{\Omega_{i}}\left(\partial_{t} e_{i}^{k}\right)+\left\|\partial_{x x} e_{i}^{k}\right\|_{L^{2}\left(\Omega_{i}\right)}^{2}\right) d s,
$$

and evaluate $\left\|\partial_{x x} e_{i}^{k}\right\|_{L^{2}\left(\Omega_{i} \times(0, t)\right)}^{2}$ by equation (4.2):

$$
\left\|\partial_{x x} e_{i}^{k}\right\|_{L^{2}\left(\Omega_{i} \times(0, t)\right)}^{2} \leq 3\left(\left\|\partial_{t t} e_{i}^{k}\right\|_{L^{2}\left(\Omega_{i} \times(0, t)\right)}^{2}+\left\|e_{i}^{k}\right\|_{L^{2}\left(\Omega_{i} \times(0, t)\right)}^{2}+\left\|f_{i}^{k}\right\|_{L^{2}\left(\Omega_{i} \times(0, t)\right)}^{2}\right),
$$


from which we deduce

$$
\left\|e_{i}^{k}\right\|_{H^{2}\left(\Omega_{i} \times(0, t)\right)}^{2} \leq 4 \int_{0}^{t}\left(E_{\Omega_{i}}\left(e_{i}^{k}\right)+E_{\Omega_{i}}\left(\partial_{t} e_{i}^{k}\right)\right) d s+3\left\|f_{i}^{k}\right\|_{L^{2}\left(\Omega_{i} \times(0, t)\right)}^{2} .
$$

There remains only in (4.12) that

$$
\begin{aligned}
& \sum_{i=1}^{I}\left(E_{\Omega_{i}}\left(e_{i}^{k}\right)+E_{\Omega_{i}}\left(\partial_{t} e_{i}^{k}\right)\right)(t)+\int_{0}^{t} \sum_{i=1}^{I}\left(E_{\partial \Omega_{i}}\left(e_{i}^{k}\right)+E_{\partial \Omega_{i}}\left(\partial_{t} e_{i}^{k}\right)\right)(s) d s \\
& \leq \int_{0}^{t} \sum_{i=1}^{I}\left(E_{\partial \Omega_{i}}\left(e_{i}^{k-1}\right)+E_{\partial \Omega_{i}}\left(\partial_{t} e_{i}^{k-1}\right)\right)(s) d s \\
& +\left[\sum_{i=1}^{I} 4 \theta_{3}\left(\sum_{\substack{|\alpha| \leq 2 \\
\alpha \neq(0,2)}}\left(\left\|\partial^{\alpha} u_{i}\right\|_{L^{2}(\Omega \times(0, T))}^{2}+\left\|\partial^{\alpha} u_{i}^{k}\right\|_{L^{2}(\Omega \times(0, T))}^{2}\right)\right)\right. \\
& \left.\times \int_{0}^{t}\left(E_{\Omega_{i}}\left(e_{i}^{k}\right)+E_{\Omega_{i}}\left(\partial_{t} e_{i}^{k}\right)\right) d s\right] \\
& +\sum_{i=1}^{I}\left(3 \theta _ { 3 } \left(\sum _ { \substack { | \alpha | \leq 2 \\
\alpha \neq ( 0 , 2 ) } } \left(\left\|\partial^{\alpha} u_{i}\right\|_{L^{2}(\Omega \times(0, T))}^{2}\right.\right.\right. \\
& \left.\left.\left.+\left\|\partial^{\alpha} u_{i}^{k}\right\|_{L^{2}(\Omega \times(0, T))}^{2}\right)\right)+\frac{1}{2}\right)\left(\left\|f_{i}^{k}\right\|_{L^{2}(\Omega \times(0, T))}^{2}+\left\|\partial_{t} f_{i}^{k}\right\|_{L^{2}(\Omega \times(0, T))}^{2}\right) .
\end{aligned}
$$

Again, as in Lemma 3.3, there exists a positive increasing function $\theta_{4}$ such that

$$
\begin{aligned}
& \left\|f_{i}^{k}(t, \cdot)\right\|_{L^{2}(\Omega)}^{2}+\left\|\partial_{t} f_{i}^{k}(t, \cdot)\right\|_{L^{2}(\Omega)}^{2} \\
& \quad \leq \theta_{4}^{2}\left(\sum_{\substack{|\alpha| \leq 2 \\
\alpha \neq(0,2)}}\left(\left\|\partial^{\alpha} u_{i}\right\|_{L^{2}(\Omega)}^{2}+\left\|\partial^{\alpha} u_{i}^{k}\right\|_{L^{2}(\Omega)}^{2}\right)\right) \sum_{\substack{|\alpha| \leq 2 \\
\alpha \neq(0,2)}}\left\|\partial^{\alpha} e_{i}^{k}\right\|_{L^{2}(\Omega)}^{2} ;
\end{aligned}
$$

the latter sum means that no term $\partial_{x x}$ are present, therefore we can bound the sum by twice the energy. Furthermore, we know that the energy of $\bar{u}$ is bounded on the interval $(0, T)$. Thus there exists a new positive increasing function $\theta_{5}$, depending on $u$, such that

$$
\begin{aligned}
& \left\|f_{i}^{k}(t, \cdot)\right\|_{L^{2}(\Omega)}^{2}+\left\|\partial_{t} f_{i}^{k}(t, \cdot)\right\|_{L^{2}(\Omega)}^{2} \\
& \quad \leq\left(\theta_{5}^{2}\left(E_{\Omega_{i}}\left(e_{i}^{k}\right)+E_{\Omega_{i}}\left(\partial_{t} e_{i}^{k}\right)\right)\left(E_{\Omega_{i}}\left(e_{i}^{k}\right)+E_{\Omega_{i}}\left(\partial_{t} e_{i}^{k}\right)\right)\right)(t) .
\end{aligned}
$$

We insert (4.14) into (4.13), and get (with a new function $\theta_{6}$ )

$$
\begin{aligned}
\sum_{i=1}^{I}\left(E_{\Omega_{i}}\left(e_{i}^{k}\right)+E_{\Omega_{i}}\left(\partial_{t} e_{i}^{k}\right)\right)(t)+\int_{0}^{t} \sum_{i=1}^{I}\left(E_{\partial \Omega_{i}}\left(e_{i}^{k}\right)+E_{\partial \Omega_{i}}\left(\partial_{t} e_{i}^{k}\right)\right)(s) d s \\
\leq \int_{0}^{t} \sum_{i=1}^{I}\left(E_{\partial \Omega_{i}}\left(e_{i}^{k-1}\right)+E_{\partial \Omega_{i}}\left(\partial_{t} e_{i}^{k-1}\right)\right)(s) d s \\
\quad+\sum_{i=1}^{I} \int_{0}^{t}\left(\theta_{6}\left(E_{\Omega_{i}}\left(e_{i}^{k}\right)+E_{\Omega_{i}}\left(\partial_{t} e_{i}^{k}\right)\right)\right)\left(E_{\Omega_{i}}\left(e_{i}^{k}\right)+E_{\Omega_{i}}\left(\partial_{t} e_{i}^{k}\right)\right)(s) d s
\end{aligned}
$$

For clarity we define

$$
E_{i n t}^{k}=\sum_{i=1}^{I}\left(E_{\Omega_{i}}\left(e_{i}^{k}\right)+E_{\Omega_{i}}\left(\partial_{t} e_{i}^{k}\right)\right), \quad E_{b}^{k}=\sum_{i=1}^{I}\left(E_{\partial \Omega_{i}}\left(e_{i}^{k}\right)+E_{\partial \Omega_{i}}\left(\partial_{t} e_{i}^{k}\right)\right),
$$


and we can rewrite (4.15) as

$$
E_{i n t}^{k}(t)+\int_{0}^{t} E_{b}^{k}(s) d s \leq \int_{0}^{t} E_{b}^{k-1}(s) d s+\int_{0}^{t} \theta_{6}\left(E_{i n t}^{k}(s)\right) E_{i n t}^{k}(s) d s .
$$

Summing in $k$, we define $\widetilde{E}_{i n t}^{K}=\sum_{k=1}^{K} E_{i n t}^{k}$, and we have

$$
\widetilde{E}_{i n t}^{K}(t)+\int_{0}^{t} E_{b}^{K}(s) d s \leq \int_{0}^{t} E_{b}^{0}(s) d s+\int_{0}^{t} \theta_{6}\left(\widetilde{E}_{i n t}^{K}(s)\right) \widetilde{E}_{\text {int }}^{K}(s) d s .
$$

Now let $C>0$. If $t$ tends to $0, \int_{0}^{t} E_{b}^{1}(s) d s+t C \theta_{6}(C)$ tends to 0 . Therefore there exists a $T_{1}$ such that $\int_{0}^{T_{1}} E_{b}^{1}(s) d s+T_{1} C \theta_{6}(C)=C$, and so we have for $t \leq T_{1}$,

$$
\widetilde{E}_{\text {int }}^{K}(t) \leq C .
$$

We conclude that $u_{i}^{k}$ exists on the time interval $\left(0, T_{1}\right)$, and that $\sum_{i=1}^{I}\left(E_{\Omega_{i}}\left(e_{i}^{k}\right)+\right.$ $\left.E_{\Omega_{i}}\left(\partial_{t} e_{i}^{k}\right)\right)$ tends to 0 when $k$ tends to infinity; the sequence $u_{i}^{k}$ converges to $\bar{u}$ on $\left(0, T_{1}\right)$ in each subdomain in the norm of energy.

\section{A finite Volume Discretization}

We use here a finite volumes scheme, which has been described in 7 for the linear one-dimensional wave equation, and extended to the nonlinear boundary value problems in the frame of absorbing boundary conditions in [11]. We restrict ourselves to uniform meshes in time and space.

5.1. Discretization of the subdomain problem (3.1). The domain $\Omega \times(0, T)$ is meshed by a rectangular grid, with uniform mesh sizes $\Delta x$ and $\Delta t$. There are $J+1$ points in space with $\Delta x=\left(a_{+}-a_{-}\right) / J$, and $N+1$ points in time, with $\Delta t=T / N$. We denote the numerical approximation to $u\left(a_{-}+j \Delta x, n \Delta t\right)$ by $U(j, n)$. We introduce the notations:

$$
\begin{aligned}
D_{t}^{+} U(j, n) & =\frac{U(j, n+1)-U(j, n)}{\Delta t}, \quad D_{t}^{-} U(j, n)=\frac{U(j, n)-U(j, n-1)}{\Delta t}, \\
D_{x}^{+} U(j, n) & =\frac{U(j+1, n)-U(j, n)}{\Delta x}, \quad D_{x}^{-} U(j, n)=\frac{U(j, n)-U(j-1, n)}{\Delta x}, \\
D_{x}^{0} U(j, n) & =\frac{U(j+1, n)-U(j-1, n)}{2 \Delta x}, \quad D_{t}^{0} U(j, n)=\frac{U(j, n+1)-U(j, n-1)}{2 \Delta t}, \\
D_{t}^{--} U(j, n) & =\frac{3 U(j, n)-4 U(j, n-1)+U(j, n-2)}{2 \Delta t}, \\
D_{t}^{-*} U(j, n) & =\left\{\begin{array}{l}
D_{t}^{--} U(j, n) \text { for } n \geq 2, \\
D_{t}^{-} U(j, n) \text { for } n=1 .
\end{array}\right.
\end{aligned}
$$

The last finite derivative in (5.1) is a second order approximation of $\partial_{t} u$, to be used in the nonlinear term, in order to design an explicit scheme.

The scheme in the interior gives

$$
\begin{aligned}
&\left(D_{t}^{+} D_{t}^{-}-D_{x}^{+} D_{x}^{-}\right) U(j, n)-f\left(U(j, n), D_{t}^{-*} U(j, n), D_{x}^{0} U(j, n)\right)=0 \\
& \text { in }[1, J-1] \times \llbracket 1, N-1 \rrbracket .
\end{aligned}
$$


We define the discrete initial value as

$$
P(j)=p\left(a_{-}+j \Delta x\right), \quad Q(j)=q\left(a_{-}+j \Delta x\right),
$$

and we obtain the initial scheme

$$
\left(D_{t}^{+}-\frac{\Delta t}{2} D_{x}^{+} D_{x}^{-}\right) U(j, 0)=Q(j)+\frac{\Delta t}{2} f\left(P(j), Q(j), D_{x}^{0} Q(j)\right), \quad \text { in }[1, J-1] .
$$

For the boundary conditions, we define the discrete boundary operators as:

$$
\begin{aligned}
B^{-}\left(f, g^{-}\right) U(0, n)= & \left(D_{t}^{0}-D_{x}^{+}+\frac{\Delta x}{2} D_{t}^{+} D_{t}^{-}\right) U(0, n) \\
& -\frac{\Delta x}{2} f\left(U(0, n), D_{t}^{-*} U(0, n), D_{x}^{+} U(0, n)\right)+g^{-}(U(0, n)), \\
B^{-}\left(f, g^{-}\right) U(0,0)= & \left(D_{t}^{+}-D_{x}^{+}+\frac{\Delta x}{\Delta t} D_{t}^{+}\right) U(0,0)-\frac{\Delta x}{\Delta t} Q(0) \\
& -\frac{\Delta x}{2} f\left(P(0), Q_{i}(0), D_{x}^{+} P(0)\right)+g^{-}(P(0)), \\
B^{+}\left(f, g^{+}\right) U(J, n)= & \left(D_{t}^{0}+D_{x}^{-}+\frac{\Delta x}{2} D_{t}^{+} D_{t}^{-}\right) U(J, n) \\
& -\frac{\Delta x}{2} f\left(U(J, n), D_{t}^{-*} U_{i}(J, n), D_{x}^{-} U(J, n)\right)+g^{+}(U(J, n)), \\
B^{+}\left(f, g^{+}\right) U(J, 0)= & \left(D_{t}^{+}+D_{x}^{-}+\frac{\Delta x}{\Delta t} D_{t}^{+}\right) U(J, 0)-\frac{\Delta x}{\Delta t} Q(J) \\
& -\frac{\Delta x}{2} f\left(P(J), Q_{i}(J), D_{x}^{-} P(J)\right)+g^{+}(P(J)) .
\end{aligned}
$$

We define the boundary data as

$$
H^{ \pm}(n)=\frac{1}{\Delta t} \int_{t_{n}-\Delta t / 2}^{t_{n}+\Delta t / 2} h^{ \pm}(\tau) d \tau, \quad 1 \leq n \leq N, H^{ \pm}(0)=\frac{2}{\Delta t} \int_{0}^{\Delta t / 2} h^{ \pm}(\tau) d \tau
$$

The discretization of problem (3.1) is now given by (5.2), (5.3), with boundary conditions

$$
B^{-}\left(f, g^{-}\right) U(0, \cdot)=H^{-}, B^{+}\left(f, g^{+}\right) U(J, \cdot)=H^{+} \text {in }[0, n \leq N \rrbracket,
$$

where the discrete boundary operators $B^{ \pm}$are given in (5.4), (5.5).

Our numerical computations indicate that this scheme is second order both in space and time.

5.2. The discrete Schwarz waveform relaxation algorithm. The equation is now discretized on each subdomain $\Omega_{i} \times(0, T), i=1, \ldots, I$ separately, using a uniform mesh with sizes $\Delta x$ and $\Delta t$. There are $J_{i}+1$ points in space and $N+1$ grid points in time in subdomain $\Omega_{i}$, with $\Delta x=\left(a_{i+1}-a_{i}\right) / J_{i}$ and $\Delta t=T / N$. We denote the numerical approximation to $u_{i}^{k}\left(a_{i}+j \Delta x, n \Delta t\right)$ on $\Omega_{i}$ at iteration step $k$ by $U_{i}^{k}(j, n)$.

The problem in domain $\Omega_{i}$ is now defined through boundary data $H_{i}^{ \pm, k-1}$, coming from the neighboring subdomains $\Omega_{i \pm 1}$. Therefore, we define the extraction 
operator from domain $\Omega_{i}$ to his neighbours as:

$$
\begin{aligned}
\widetilde{B}^{+}\left(f, g^{+}\right) U_{i}(0, n)= & \left(D_{t}^{0}+D_{x}^{+}-\frac{\Delta x}{2} D_{t}^{+} D_{t}^{-}\right) U_{i}(0, n) \\
& +\frac{\Delta x}{2} f\left(U_{i}(0, n), D_{t}^{-*} U_{i}(0, n), D_{x}^{+} U_{i}(0, n)\right)+g^{+}\left(U_{i}(0, n)\right), \\
\widetilde{B}^{+}\left(f, g^{+}\right) U_{i}(0,0)= & \left(D_{t}^{+}+D_{x}^{+}-\frac{\Delta x}{\Delta t} D_{t}^{+}\right) U_{i}(0,0) \\
& +\frac{\Delta x}{\Delta t} Q_{i}(0)+\frac{\Delta x}{2} f\left(P_{i}(0), Q_{i}(0), D_{x}^{+} P_{i}(0)\right)+g^{+}\left(P_{i}(0),\right. \\
(5.9) & +\frac{\Delta x}{2} f\left(U_{i}\left(J_{i}, n\right), D_{t}^{-*} U_{i}\left(J_{i}, n\right), D_{x}^{-} U_{i}\left(J_{i}, n\right)\right)+g^{-}\left(U_{i}\left(J_{i}, n\right)\right), \\
\widetilde{B}^{-}\left(f, g^{-}\right) U_{i}\left(J_{i}, n\right)= & \left(D_{t}^{0}-D_{x}^{-}-\frac{\Delta x}{2} D_{t}^{+} D_{t}^{-}\right) U_{i}\left(J_{i}, n\right) \\
& \left(D_{t}^{+}-D_{x}^{-}-\frac{\Delta x}{\Delta t} D_{t}^{+}\right) U_{i}\left(J_{i}, 0\right)+\frac{\Delta x}{\Delta t} Q_{i}\left(J_{i}\right) \\
& +\frac{\Delta x}{2} f\left(P_{i}\left(J_{i}\right), Q_{i}\left(J_{i}\right), D_{x}^{-} P_{i}\left(J_{i}\right)\right)+g^{-}\left(P_{i}\left(J_{i}\right)\right) .
\end{aligned}
$$

The discrete Schwarz waveform relaxation algorithm on subdomains $\Omega_{i}, i=$ $1, \ldots, I$ is defined as follows. An initial guess $\left\{H_{i}^{0, \pm}\right\}_{1 \leq i \leq I}$ is given. For $k \geq 1$, we solve

$$
\begin{aligned}
& {\left[\begin{array}{l}
\left(D_{t}^{+} D_{t}^{-}-D_{x}^{+} D_{x}^{-}\right) U_{i}^{k}=f\left(U_{i}^{k}, D_{t}^{-*} U_{i}^{k}, D_{x}^{0} U_{i}^{k}\right) \text { in }\left[1, J_{i}-1 \rrbracket \times \llbracket 1, N \rrbracket,\right. \\
U_{i}^{k}(\cdot, 0)=P_{i}, \quad\left(D_{t}^{+}-\frac{\Delta t}{2} D_{x}^{+} D_{x}^{-}\right) U_{i}^{k}(\cdot, 0)=Q_{i}+\frac{\Delta t}{2} f\left(P_{i}, Q_{i}, D_{x}^{0} Q_{i}\right) \text { in } \llbracket 1, J_{i}-1 \rrbracket, \\
B^{-}\left(f, g^{-}\right) U_{i}^{k}(0, \cdot)=H_{i}^{-, k-1}, B^{+}\left(f, g^{+}\right) U_{i}^{k}\left(J_{i}, \cdot\right)=H_{i}^{+, k-1} \text { in }[0, n \leq N \rrbracket, \\
H_{i}^{-, k}=\widetilde{B}^{-}\left(f, g^{-}\right) U_{i-1}^{k}\left(J_{i-1}, \cdot\right), H_{i}^{+, k}=\widetilde{B}^{+}\left(f, g^{+}\right) U_{i+1}^{k}(0, \cdot) \text { in }[0, n \leq N \rrbracket .
\end{array}\right.}
\end{aligned}
$$

As in the continuous algorithm, we set $U_{0}^{k} \equiv 0$ and $U_{I+2}^{k} \equiv 0$.

We denote by $\bar{U}$ the discrete approximation of problem (2.4), obtained by solving (5.2), (5.3), (5.7) on $\Omega=(a, b)$ with $J=\sum_{i=1}^{I} J_{i}$ intervals of length $\Delta x$, and $H^{ \pm}=$ 0 . Each subproblem is an explicit scheme, thus has a unique solution. Therefore the Schwarz waveform Relaxation Algorithm is well defined. If it converges, the limit in each subdomain is denoted by $V_{i}$. It satisfies the same scheme as $\bar{U}$ at initial time, in the interior and on the exterior boundaries. At point $a_{i}$ it satisfies for any $n \geq 0$,

$$
\begin{aligned}
B^{-}\left(f, g^{-}\right) V_{i}(0, n) & =\widetilde{B}^{-}\left(f, g^{-}\right) V_{i-1}\left(J_{i-1}, n\right), B^{+}\left(f, g^{+}\right) V_{i-1}\left(J_{i-1}, n\right) \\
& =\widetilde{B}^{+}\left(f, g^{+}\right) V_{i}(0, n) .
\end{aligned}
$$

Theorem 5.1. Suppose that $f$ is affine in the third variable $\partial_{x} u$. Then, if the discrete algorithm converges, it converges to the discrete approximation $\bar{U}$ of problem (2.4).

Proof. Since $p$ is in $H^{2}(\Omega)$ and $q$ is in $H^{1}(\Omega)$, they are both continuous and we have for any $i, V_{i}(0,0)=P_{i}(0)=V_{i-1}\left(J_{i-1}, 0\right)=P_{i-1}\left(J_{i-1}\right)=p\left(a_{i}\right)$, and $Q_{i-1}\left(J_{i-1}\right)=$ 
$Q_{i}(0)=q\left(a_{i}\right)$. We write the transmission conditions (5.11) for $n=0$. The nonlinear terms containing $g^{ \pm}$on both sides cancel out, and we have

$$
\begin{aligned}
& (5.12) \quad\left(D_{t}^{+}-D_{x}^{+}+\frac{\Delta x}{\Delta t} D_{t}^{+}\right) V_{i}(0,0)-\frac{\Delta x}{\Delta t} q\left(a_{i}\right)-\frac{\Delta x}{2} f\left(p\left(a_{i}\right), q\left(a_{i}\right), D_{x}^{+} p\left(a_{i}\right)\right) \\
& =\left(D_{t}^{+}-D_{x}^{-}-\frac{\Delta x}{\Delta t} D_{t}^{+}\right) V_{i-1}\left(J_{i-1}, 0\right)+\frac{\Delta x}{\Delta t} q\left(a_{i}\right)+\frac{\Delta x}{2} f\left(p\left(a_{i}\right), q\left(a_{i}\right), D_{x}^{-} p\left(a_{i}\right)\right),
\end{aligned}
$$

$$
\begin{aligned}
&\left(D_{t}^{+}+D_{x}^{+}-\frac{\Delta x}{\Delta t} D_{t}^{+}\right) V_{i}(0,0)+\frac{\Delta x}{\Delta t} q\left(a_{i}\right)+\frac{\Delta x}{2} f\left(p\left(a_{i}\right), q\left(a_{i}\right), D_{x}^{+} p\left(a_{i}\right)\right) \\
&\left(D_{t}^{+}+D_{x}^{-}+\frac{\Delta x}{\Delta t} D_{t}^{+}\right) V_{i-1}\left(J_{i-1}, 0\right)-\frac{\Delta x}{\Delta t} q\left(a_{i}\right)-\frac{\Delta x}{2} f\left(p\left(a_{i}\right), q\left(a_{i}\right), D_{x}^{-} p\left(a_{i}\right)\right) .
\end{aligned}
$$

Adding (5.12) and (5.13) yields $D_{t}^{+} V_{i}(0,0)=D_{t}^{+} V_{i-1}\left(J_{i-1}, 0\right)$, and hence $V_{i}(0,1)$ $=V_{i-1}\left(J_{i-1}, 1\right)$. We define now $\tilde{V}(j, n)$ for $0 \leq j \leq J$ as $\tilde{V}(\tilde{j}, n)=V_{i}(j, n)$ if $\tilde{j} \Delta x$ $=a_{i}+j \Delta x$, with $1 \leq j \leq J_{i}$. With the assumption on $f$, since $D_{x}^{0}=\left(D_{x}^{+}+D_{x}^{-}\right) / 2$, we can rewrite (5.12) as

$$
\left(D_{x}^{+}-D_{x}^{-}-2 \frac{\Delta x}{\Delta t} D_{t}^{+}\right) \tilde{V}\left(\tilde{j}_{i}, 0\right)+2 \frac{\Delta x}{\Delta t} q\left(a_{i}\right)+\Delta x f\left(p\left(a_{i}\right), q\left(a_{i}\right), D_{x}^{0} p\left(a_{i}\right)\right)=0,
$$

with $\tilde{j}_{i}=J_{1}+\cdots+J_{i}$, which, when multiplying by $-\frac{\Delta t}{2 \Delta x}$, proves that $\tilde{V}$ is the solution of (5.3) at any point, and therefore $U$ and $\tilde{V}$ coincide at time 0 and 1. A simple recursion with the explicit schemes now proves that, for any $n$, for any $i$, $V_{i}(0, n)=V_{i-1}\left(J_{i-1}, n\right)$, and therefore $U=\tilde{V}$.

Remark. The assumption on $f$ in Theorem 5.1 is fulfilled when $f\left(u, u_{t}, u_{x}\right)=$ $f_{1}(u)+f_{2}(u) u_{t}+f_{3}(u) u_{x}$.

\section{Convergence of the Discrete Algorithm}

According to Theorem 5.1, we suppose that $f$ is affine in $\partial_{x} u$. We introduce the linear transmission operators defined by

$$
T^{ \pm}=B^{ \pm}(0,0), \quad \widetilde{T}^{ \pm}=\widetilde{B}^{ \pm}(0,0) .
$$

We note $\bar{U}_{i}=\bar{U} / \Omega_{i}$. With these notations, the error $\bar{U}_{i}^{k}=U_{i}^{k}-\bar{U}_{i}$ is the solution of the linear problem

$$
\left(D_{t}^{+} D_{t}^{-}-D_{x}^{+} D_{x}^{-}+1\right) \bar{U}_{i}^{k}=F_{i}^{k}, \quad \text { in }\left[1, J_{i}-1\right] \times[1, N]
$$

with the initial value $\bar{U}_{i}^{k}(j, 1)=\bar{U}_{i}^{k}(j, 0)=0$ and the transmission conditions

$$
\begin{aligned}
T^{-} \bar{U}_{i}^{k}(0, \cdot)+G_{i}^{-, k}=\widetilde{T}^{-} \bar{U}_{i-1}^{k-1}\left(J_{i-1}, \cdot\right)+\widetilde{G}_{i-1}^{-, k-1}, & \text { in }[0, N], \\
T^{+} \bar{U}_{i}^{k}\left(J_{i}, \cdot\right)+G_{i}^{+, k}=\widetilde{T}^{+} \bar{U}_{i+1}^{k-1}(0, \cdot)+\widetilde{G}_{i+1}^{+, k-1}, & \text { in }[0, N] .
\end{aligned}
$$

The remainders are given by

$$
\begin{aligned}
& F_{i}^{k}=f\left(U_{i}^{k}, D_{t}^{-*} U_{i}^{k}, D_{x}^{0} U_{i}^{k}\right)-f\left(\bar{U}_{i}, D_{t}^{-*} U_{i}^{k}, D_{x}^{0} \bar{U}_{i}\right)+\bar{U}_{i}^{k} \text { for } n \geq 1, \\
& G_{i}^{-, k}=-\frac{\Delta x}{2} f_{i}^{k}(0, \cdot)+g^{-}\left(U_{i}^{k}(0, \cdot)\right)-g\left(\bar{U}_{i}(0, \cdot)\right), \\
& \widetilde{G}_{i-1}^{-, k}=\frac{\Delta x}{2} f_{i-1}^{k}\left(J_{i-1}, \cdot\right)+g^{-}\left(\bar{U}_{i-1}^{k}\left(J_{i-1}, \cdot\right)\right)-g^{-}\left(\bar{U}_{i-1}\left(J_{i-1}, \cdot\right)\right), \\
& G_{i}^{+, k}=-\frac{\Delta x}{2} f_{i}^{k}\left(J_{i}, \cdot\right)+g^{+}\left(\bar{U}_{i}^{k}\left(J_{i}, \cdot\right)\right)-g^{+}\left(\bar{U}_{i}\left(J_{i}, \cdot\right)\right), \\
& \widetilde{G}_{i+1}^{+, k}=\frac{\Delta x}{2} f_{i+1}^{k}(0, \cdot)+g^{+}\left(\bar{U}_{i+1}^{k}(0, \cdot)\right)-g^{+}\left(\bar{U}_{i+1}(0, \cdot)\right),
\end{aligned}
$$


and $G_{i}^{ \pm, k}(0)=0, \widetilde{G}_{i}^{ \pm, k}(0)=0$. For $n=0$, the centered derivative in $x$ is replaced in the expression of $F_{i}^{k}$ by a forward or backward derivative.

We define now a discrete energy as follows. We consider sequences of the form $V=\{V(j)\}_{0 \leq j \leq J}$ in $\mathbb{R}^{J+1}$, and we define a bilinear form on $\mathbb{R}^{J+1}$ by

$$
a_{\Delta}(V, W)=\Delta x\left(\sum_{j=1}^{J} D_{x}^{-}(V)(j) \cdot D_{x}^{-}(W)(j)+\sum_{j=1}^{J-1} V(j) W(j)\right) .
$$

For a mesh function $V$ of time and space, we define

$$
\begin{aligned}
& E_{K}(V)(n)=\frac{\Delta x}{2} \sum_{j=1}^{J-1}\left(\left(D_{t}^{-} V(j, n)\right)^{2}+\left(D_{t}^{-} V(j, n+1)\right)^{2}\right), \\
& E_{P}(V)(n)=a_{\Delta}(V(\cdot, n), V(\cdot, n-1)), \\
& E=E_{K}+E_{P} .
\end{aligned}
$$

The quantity $E_{K}$ is a discrete kinetic energy, and $E_{P}$ is a discrete potential energy. The following lemma gives a lower bound for $E$ under a CFL condition, and hence shows that $E$ is then indeed an energy. The proof is classical ([7]) and is omitted here.

Lemma 6.1. For any $n \geq 1$, we have

$$
E(V)(n) \geq\left(1-\frac{\Delta t^{2}}{\Delta x^{2}}-\frac{\Delta t^{2}}{4}\right) E_{K}(V)(n) .
$$

Hence, under the CFL condition

$$
\frac{\Delta t^{2}}{\Delta x^{2}}+\frac{\Delta t^{2}}{4}<1
$$

$E$ is bounded from below by an energy.

The following energy estimate is obtained by a discrete integration by parts:

Lemma 6.2. For any $V$ solution of

$$
\left(D_{t}^{+} D_{t}^{-}-D_{x}^{+} D_{x}^{-}+1\right) V=F, \quad 1 \leq j \leq J-1,1 \leq n \leq N,
$$

we have for any $n \geq 1$,

$$
\begin{aligned}
& E(V)(n)-E(V)(n-1)+\frac{\Delta t}{2}\left[\left(\widetilde{T}^{+} V(0, n)\right)^{2}+\left(\widetilde{T}^{-} V(J, n)\right)^{2}\right] \\
& =\frac{\Delta t}{2}\left[\left(T^{-} V(0, n)\right)^{2}+\left(T^{+} V(J, n)\right)^{2}\right]+2 \Delta t \Delta x \sum_{j=1}^{J} F(j, n) D_{t}^{0} V(j, n),
\end{aligned}
$$

and for $n=0$,

$$
\begin{aligned}
& E_{K}(U)(0)+E(U)(0)+\frac{\Delta t}{4}\left[\left(\widetilde{T}^{+} U(0,0)\right)^{2}+\left(\widetilde{T}^{-} U(J+1,0)\right)^{2}\right] \\
& \quad=\frac{\Delta t}{4}\left[\left(T^{-} U(0,0)\right)^{2}+\left(T^{+} U(J, 0)\right)^{2}\right]+a_{\Delta}(P, P)+2 \Delta x \sum_{j=1}^{J} Q(j) D_{t}^{+} U(j, 0) .
\end{aligned}
$$

These estimates are obtained by multiplying (6.8) with $D_{t}^{0} V(j, n)$ and integrating by parts [7.

We now state the main result of this section. 
Theorem 6.3. Suppose that $f$ is affine with respect to $\partial_{x} u$. Defining the quantities (6.11)

$$
\begin{aligned}
R_{i}^{k}(n)= & \widetilde{G}_{i}^{+, k}(n)\left(\widetilde{G}_{i}^{+, k}(n)+2 \widetilde{T}^{+} \bar{U}_{i}^{k}(0, n)\right)+\widetilde{G}_{i}^{-, k}(n)\left(\widetilde{G}_{1}^{-, k}(n)+2 \widetilde{T}^{-} \bar{U}_{i}^{k}\left(J_{i}, n\right)\right) \\
& -G_{i}^{-, k}(n)\left(G_{i}^{-, k}(n)+2 T^{-} \bar{U}_{i}^{k}(0, n)\right)-G_{i}^{+, k}(n)\left(G_{i}^{+, k}(n)+2 T^{+} \bar{U}_{i}^{k}\left(J_{i}, n\right)\right),
\end{aligned}
$$

and assuming that there exists a positive constant $M$ such that for any iteration number $K$, any domain $\Omega_{i}$ and any discrete time $n$, the following estimate holds:

$$
2 \Delta x \sum_{j=1}^{J} F_{i}^{k}(j, n) D_{t}^{0} \bar{U}_{i}^{k}(j, n)+\frac{1}{2} R_{i}^{k}(n) \leq M E\left(\bar{U}_{i}^{k}\right)(n),
$$

then, for $\Delta t$ sufficiently small, the discrete Schwarz algorithm converges in the energy norm.

Remark. Assumption (6.12) is technical, and will be proved in Corollary 6.4 in a special case.

Proof. We apply (6.9), (6.10) to $\bar{U}_{i}^{k}$. Since the initial data vanish, every term in (6.10) vanishes. Thus $E\left(\bar{U}_{i}^{k}\right)(0)=0$, and we rewrite (6.9) as

$$
\begin{aligned}
E\left(\bar{U}_{i}^{k}\right)(n)-E\left(\bar{U}_{i}^{k}\right)(n-1) & \\
& +\frac{\Delta t}{2}\left[\left(\widetilde{T}^{+} \bar{U}_{i}^{k}(0, n)+\widetilde{G}_{i}^{+, k}(n)\right)^{2}+\left(\widetilde{T}^{-} \bar{U}_{i}^{k}\left(J_{i}, n\right)+\widetilde{G}_{i}^{-, k}(n)\right)^{2}\right] \\
= & \frac{\Delta t}{2}\left[\left(T^{-} \bar{U}_{i}^{k}(0, n)+G_{i}^{-, k}(n)\right)^{2}+\left(T^{+} \bar{U}_{i}^{k}\left(J_{i}, n\right)+G_{i}^{+, k}(n)\right)^{2}\right] \\
& +2 \Delta t \Delta x \sum_{j=1}^{J_{i}} f_{i}^{k}(j, n) D_{t}^{0} \bar{U}_{i}^{k}(j, n)+\frac{\Delta t}{2} R_{i}^{k}(n) .
\end{aligned}
$$

We now insert the transmission conditions (6.2), translate the indices in the righthand side, and add the contributions of all subdomains. We define a total internal energy and a total boundary energy as

$$
\begin{aligned}
E_{I}^{k}(n) & =\sum_{i=1}^{I} E\left(\bar{U}_{i}^{k}\right)(n), E_{B}^{k}(n) \\
& =\frac{\Delta t}{2} \sum_{i}\left[\left(\widetilde{T}^{+} \bar{U}_{i}^{k}(0, n)+\widetilde{G}_{i}^{+, k}(n)\right)^{2}+\left(\widetilde{T}^{-} \bar{U}_{i}^{k}\left(J_{i}, n\right)+\widetilde{G}_{i}^{-, k}(n)\right)^{2}\right] .
\end{aligned}
$$

With these notations we can write

$$
\begin{aligned}
& E_{I}^{k}(n)-E_{I}^{k}(n-1)+E_{B}^{k}(n)=E_{B}^{k-1}(n) \\
& \quad+\sum_{i=1}^{I}\left[2 \Delta t \Delta x \sum_{j=1}^{J_{i}} f_{i}^{k}(j, n) D_{t}^{0} \bar{U}_{i}^{k}(j, n)+\frac{\Delta t}{2} R_{i}^{k}(n)\right] .
\end{aligned}
$$

We now sum up (6.14) for $1 \leq k \leq K$, and define $\hat{E}_{I}^{K}(n)=\sum_{k=1}^{K} E_{I}^{k}(n)$ :

$$
\begin{aligned}
& \hat{E}_{I}^{K}(n)-\hat{E}_{I}^{K}(n-1)+E_{B}^{K}(n)=E_{B}^{0}(n) \\
& \quad+\Delta t \sum_{k=1}^{K} \sum_{i=1}^{I}\left[2 \Delta x \sum_{j=1}^{J_{i}} f_{i}^{k}(j, n) D_{t}^{0} \bar{U}_{i}^{k}(j, n)+\frac{1}{2} R_{i}^{k}(n)\right] .
\end{aligned}
$$

Under assumption (6.12) we deduce that

$$
\hat{E}_{I}^{K}(n)-\hat{E}_{I}^{K}(n-1) \leq F_{0}(n)+M \Delta t \hat{E}_{I}^{K}(n) .
$$


The recursive inequality is easy to solve. For $\Delta t$ sufficiently small, $M \Delta t<1$, and since $\hat{E}_{K}(0)=0$, we get

$$
\hat{E}_{I}^{K}(n) \leq e^{M n \Delta t} \sum_{p=1}^{n} F_{0}(p) \leq e^{M T} \sum_{p=1}^{N} F_{0}(p) .
$$

This proves that $\sup _{n \leq N} \hat{E}_{I}^{K}(n)$ is bounded as $K$ tends to infinity. Therefore we have

$$
\forall n \leq N, \sum_{i=1}^{I} E\left(U_{i}^{k}-\bar{U}\right)(n) \rightarrow 0 \text { as } k \rightarrow+\infty,
$$

which concludes the proof.

We are able to prove the assumption (6.12) in the case of the linear transmission conditions.

Corollary 6.4. Suppose that $f$ is affine with respect to $\partial_{x} u$. For $\Delta t$ sufficiently small, there exists a time $T$ such that the discrete Schwarz waveform relaxation algorithm (5.10) with linear transmission conditions (i.e. $g^{ \pm}=0$ ) converges to the discrete approximation $\bar{U}$ of problem (2.4) on $(0, T)$.

Proof. Here the remainder $R_{i}^{k}(n)$ reduces to

$$
R_{i}^{k}(n)=2 \Delta x\left(-f_{i}^{k}(0, n) D_{t}^{0} \bar{U}_{i}^{k}(0, n)+f_{i}^{k}\left(J_{i}, n\right) D_{t}^{0} \bar{U}_{i}^{k}\left(J_{i}, n\right)\right),
$$

and the estimate (6.12) amounts to proving that for any $k \leq K, n \leq N$,

$$
\Delta x \sum_{j=0}^{J_{i}} f_{i}^{k}(j, n) D_{t}^{0} \bar{U}_{i}^{k}(j, n) \leq M_{1} E\left(\bar{U}_{i}^{k}\right)(n) .
$$

If $f$ is globally Lipschitz in both variables, this is merely an application of the discrete Cauchy-Schwarz lemma.

\section{NumericAl RESUlts}

7.1. Remarks on overlapping versus nonoverlapping Schwarz waveform relaxation algorithms and variants. The original Schwarz algorithm, designed for elliptic equations, uses overlapping subdomains $\left(\Omega_{i}=\left(a_{i}, b_{i}\right)\right.$ for $1 \leq i \leq I$, with $\left.a_{i}<a_{i+1}<b_{i}<b_{i+1}\right)$, with an exchange of Dirichlet data on the boundary, and the convergence factor depends on the overlap [10, 8. For hyperbolic problems, due to the finite speed of propagation, it is easy to see that this "classical" algorithm converges in a finite number of iterations, given by $N=\lceil c T / L\rceil$ where $c$ is the wave speed, and $L$ the size of the overlap. In the linear case, absorbing transmission conditions have the same property, but the convergence is drastically improved, even without overlap. In one dimension, for sufficiently small $T$, only two iterations are needed for convergence, independently of the number of subdomains, 7, and in two dimensions, the absorbing transmission conditions with optimized coefficients can reduce the error after two iterations by a factor 20 [5].

In the nonlinear case, we have theoretical convergence results on the linear algorithm for sufficiently small $T$. However, we will see that the linear algorithm outperforms the classical one on large time intervals as well, and that the nonlinear algorithm performs even better. 
Our experiments concern the space domain $\Omega=[0,4]$, simulating $\mathbb{R}$ with linear absorbing boundary conditions at each boundary. The time interval is $[0,2] . \Omega$ is divided in two subdomains. The initial value is supported in $(0,2)$, with $p(x)=$ $x^{3}(2-x)^{3}$, the initial velocity is $q(x)=3 x^{2}(2-x)^{2}(x-1)$. This is a good test since the solution is supported in the first subdomain at $t=0$ and escapes in the second domain before the end of the computation.

Note that the Schwarz algorithm can be viewed as a fixed point algorithm applied to the interface problems

$$
\left(H_{1}^{+}, H_{2}^{-}\right) \mapsto\left(\widetilde{B}^{+} U_{2}(0, \cdot), \widetilde{B}^{-} U_{1}\left(J_{1}, \cdot\right)\right)=\mathcal{A}\left(H_{1}^{+}, H_{2}^{-}\right) .
$$

In all cases, the stopping criterion in the algorithm will be on the residual res ${ }^{k}$ for $\mathcal{A}$. We also compute the exact discrete solution in $\Omega$, and measure the discrete global error $E^{k}$ in $L^{\infty}\left(0, T, L^{2}(\Omega)\right)$.

7.2. The classical overlapping Schwarz algorithm. In this case, $\Omega_{1}=(0,2+L)$ and $\Omega_{2}=(2,4)$. The stopping criterion pertains to the residual for the interface problem:

$$
r e s^{k}=\left(\left\|\left(u_{1}^{k+1}-u_{1}^{k}\right)(2+L, \cdot)\right\|_{L^{2}}^{2}+\left\|\left(u_{2}^{k+1}-u_{2}^{k}\right)(2, \cdot)\right\|_{L^{2}}^{2}\right)^{1 / 2} .
$$

We run the computation until the residual $r e s^{k}$ is equal to zero. The theoretical minimal number of iterations for the discrete algorithm is $N_{t h}^{d}=\left\lceil\frac{\Delta x}{\Delta t} \frac{T}{L}\right\rceil$, while the continuous one is $N_{t h}^{c}=\left\lceil\frac{T}{L}\right\rceil$.

We start with the nonlinear term $f\left(u, u_{t}, u_{x}\right)=u^{3}$. Table 1 gives the number of iterations $N_{\text {comp }}$ needed to achieve convergence (i.e. the error is zero), together with $N_{t h}^{d}$ and $N_{t h}^{c}$, for a fixed overlap equal to eight grid points. $\Delta t$ and $\Delta x$ vary with $\Delta t / \Delta x$ kept constant, such as to fulfill the CFL condition. The speed of convergence is a decreasing function of the stepsize. It is in accordance with the discrete theoretical convergence speed for large stepsize, and with the theoretical continuous speed for small stepsize. This is due to the fact that the smaller the stepsizes are, the closer the discrete algorithm is to the continuous algorithm.

Table 2 shows the convergence behavior of the algorithm for fixed stepsize, as a function of the overlap. The number of iterations needed to reach convergence decreases with the size of the overlap, and the behavior is similar to the continuous one for fine mesh, to the discrete one for rougher mesh.

We show on Figure 1 the convergence history of the classical Schwarz algorithm for various values of the mesh size and an overlap equal to eight grid points. We

TABLE 1. Number of iterations to achieve convergence for the classical Schwarz algorithm, $L=8 \Delta x$

\begin{tabular}{|c|cccc|}
\hline$\Delta x$ & $2 / 100$ & $1 / 100$ & $1 / 200$ & $1 / 400$ \\
\hline$\Delta t$ & $2 / 120$ & $1 / 120$ & $1 / 240$ & $1 / 480$ \\
\hline$N_{\text {comp }}$ & 16 & 29 & 54 & 105 \\
\hline$N_{t h}^{d}$ & 16 & 31 & 61 & 121 \\
\hline$N_{t h}^{c}$ & 13 & 26 & 51 & 101 \\
\hline
\end{tabular}


TABLE 2. Number of iterations to achieve convergence for the classical Schwarz algorithm as a function of the overlap

\begin{tabular}{|c|ccccc|}
\hline overlap $L$ & $\Delta x$ & $2 \Delta x$ & $4 \Delta x$ & $8 \Delta x$ & $16 \Delta x$ \\
\hline$N_{\text {comp }}$ & 220 & 111 & 56 & 29 & 15 \\
\hline$N_{t h}^{d}$ & 241 & 121 & 61 & 31 & 16 \\
\hline$N_{t h}^{c}$ & 201 & 101 & 51 & 26 & 13 \\
\hline
\end{tabular}

$\Delta x=1 / 100, \Delta t=1 / 120$

\begin{tabular}{|c|ccccc|}
\hline overlap $L$ & $\Delta x$ & $2 \Delta x$ & $4 \Delta x$ & $8 \Delta x$ & $16 \Delta x$ \\
\hline$N_{\text {comp }}$ & 61 & 31 & 16 & 9 & 5 \\
\hline$N_{t h}^{d}$ & 61 & 31 & 16 & 8 & 4 \\
\hline$N_{t h}^{c}$ & 51 & 26 & 13 & 7 & 4 \\
\hline
\end{tabular}

$\Delta x=4 / 100, \Delta t=4 / 120$

check in each case that the error $E^{k}$ vanishes together with the residual. Furthermore, we can see that the error decays very slowly for many iterations, and reaches zero in a few iterations, independently of the mesh size (three or four in all cases). The behaviour is very similar to what happens for the linear wave equation: only the finite speed of propagation produces convergence, which takes place when the signal has left the domain. The algorithm behaves similarly for other nonlinearities.
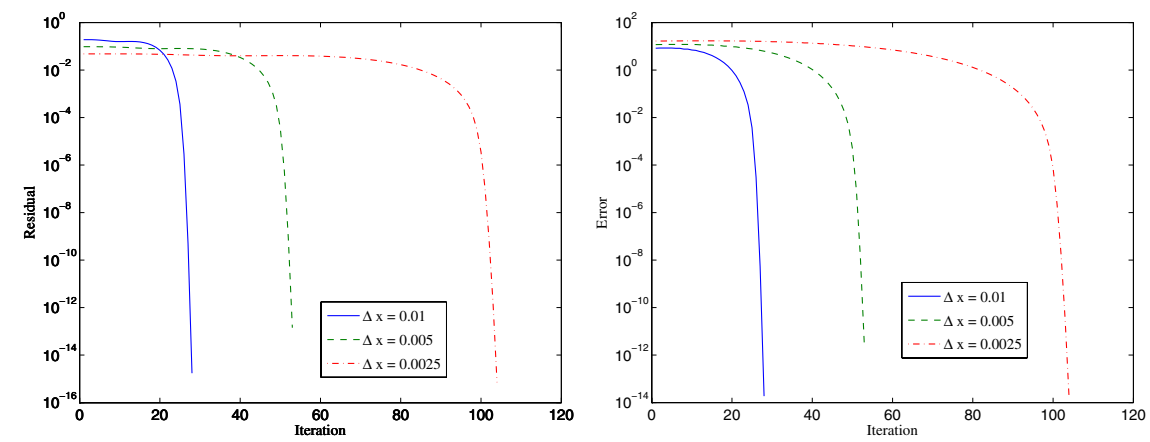

FiguRE 1. Variation of the residual $\operatorname{res}^{k}$ (left) and the error $E^{k}$ (right) as a function of the iteration number $k$.

7.3. The nonlinear nonoverlapping Schwarz algorithms. We will see in this section that our strategy greatly improves the performances of the classical Schwarz algorithm. Note that, whereas the classical algorithm only converges in the presence of an overlap, and the smaller the overlap, the slower the convergence, our algorithms are run without overlap.

Here the residual is given by

$$
r e s^{k}=\left(\left\|\left(G^{+, k+1}-G^{+, k}\right)\left(a_{1}, \cdot\right)\right\|_{L^{2}}^{2}+\left\|\left(G^{-, k+1}-G_{2}^{-, k}\right)\left(a_{1}, \cdot\right)\right\|_{L^{2}}^{2}\right)^{1 / 2} .
$$



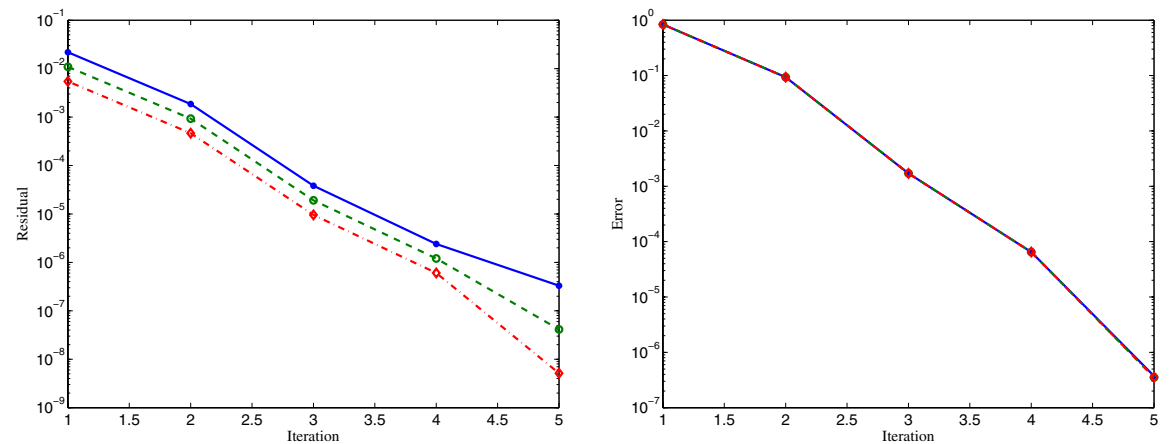

FiguRE 2. $f=u^{3}$. Left residual, right: error. $*: \Delta x=1 / 100$, $\Delta t=1 / 120$, o: $\Delta x=1 / 200, \Delta t=1 / 240, \diamond: \Delta x=1 / 400$, $\Delta t=1 / 480$.
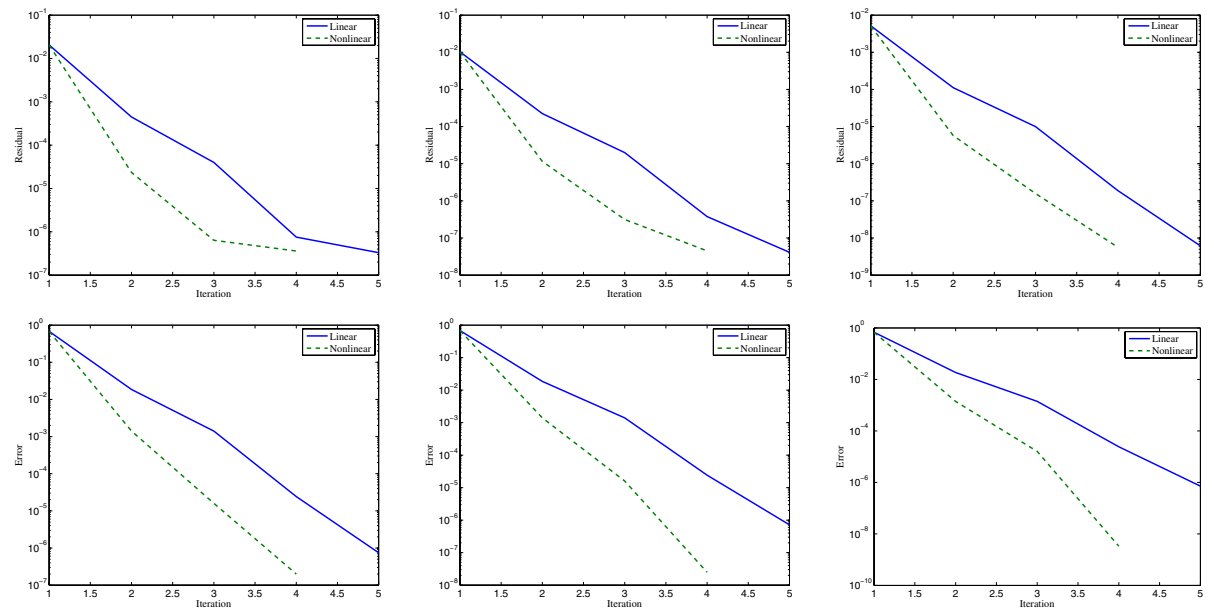

$\Delta x=1 / 100, \Delta t=1 / 120$

$\Delta x=1 / 200, \Delta t=1 / 240$

$\Delta x=1 / 400, \Delta t=1 / 480$

Figure 3. $f=u^{2} u_{x}$. Top: residual, bottom: error. Solid: linear, dash: nonlinear.

We start with $f=u^{3}$. In this case the transmission operators are the same and linear, since $g^{ \pm}=0$. We have proved in Corollary 6.4 that there exists a final time $T$ for which the discrete algorithm is convergent.

In the forthcoming computations, the theoretical and numerical data are the same as before. Figure 2 plots the convergence history for various mesh sizes. The computation is stopped as soon as the residual reaches $0.510^{-7}$. We see that the algorithm converges very rapidly, independently of the mesh size.

We consider now the case $f=u^{2} u_{x}$, and nonlinear transmission conditions, i.e. $g^{ \pm}(u)= \pm u^{3} / 6$. In Figure 3, we plot the convergence history for various mesh sizes. The computation is stopped as soon as the residual reaches $0.510^{-7}$. Both the linear and the nonlinear transmission conditions behave very well. The 


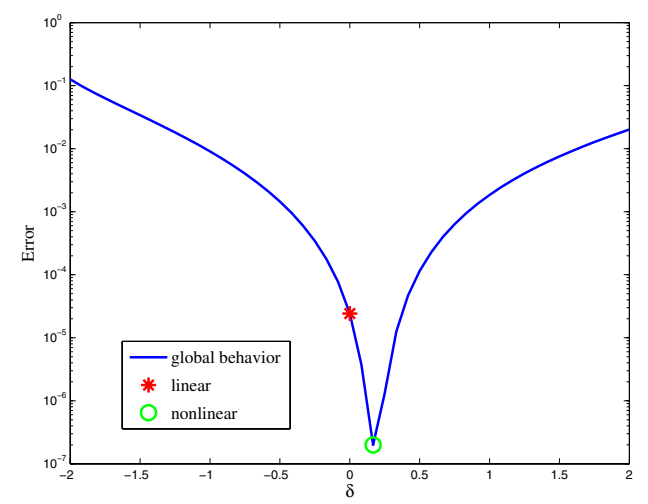

Figure 4. $f=u^{2} u_{x}$. Variations of the error as a function of the nonlinearity coefficient $\delta$.

convergence takes place in five iterations with the linear transmission condition, in four iterations with the nonlinear one.

We also can vary the nonlinearities in the transmission conditions, we use a real parameter $\delta$, and $g^{ \pm}= \pm \delta u^{3}$. The nonlinear strategy corresponds to $\delta=1 / 6$, whereas the linear one is obtained for $\delta=0$. We draw in Figure 4 the error curves after three iterations for the same initial values as before, the mesh sizes are $\Delta x=$ $1 / 100, \Delta t=1 / 120$. We observe that the nonlinear strategy corresponds precisely to the optimal numerical value of the parameter $\delta$, validating the high frequency approach. We have carried out the same computations in the case $f=u^{2} u_{t}$. We do not display the results here since they are very similar.

\section{Conclusion}

We have presented a linear and a nonlinear Schwarz waveform relaxation algorithm without overlap for the semilinear wave equation. On the continuous level, we proved the convergence for sufficiently small time intervals. We designed a discrete algorithm, in such a way that, if convergent, the algorithm converges to the discrete solution in the whole domain, which we prove when the nonlinearity is affine in $\partial_{x} u$. In that case we proved the convergence for the linear transmission condition. Numerical experiments highlight the fast convergence to the discrete full domain solution in a large time domain in both linear and nonlinear strategies, without overlap. Furthermore, we have shown that our nonlinear transmission conditions give optimal results within a large class of transmission conditions.

\section{ACKNOWLEDGMENT}

The authors are very grateful to Professor Martin Gander from Genève University whose matlab scripts were a basis for the present work.

\section{REFERENCES}

1. J. M. Bony, Calcul symbolique et propagation des singularités pour les équations aux dérivées partielles non linéaires, Ann. Sci. Ec. Norm. Sup 4 ème série 14 (1981), 209-246. MR631751 (84h:35177)

2. J. Y. Chemin, Fluides parfaits incompressibles, Astérisque 230, 1995. MR1340046|(97d:76007) 
3. B. Engquist and A. Majda, Radiation boundary conditions for acoustic and elastic calculations, Comm. Pure Appl. Math. 32 (1979), 313-357. MR517938(80e:76041)

4. M. J. Gander and C. Rohde, Overlapping Schwarz waveform relaxation for convection dominated nonlinear conservation laws, SIAM Journal on Scientific Computing 27 (2005), no. 2, 415-439. MR2202227 (2006i:35234)

5. M. J. Gander and L. Halpern, Absorbing boundary conditions for the wave equation and parallel computing, Math. of Comp. 74 (2004), no. 249, 153-176. MR2085406 (2005h:65158)

6. M. J. Gander, L. Halpern, and F. Nataf, Optimal convergence for overlapping and nonoverlapping Schwarz waveform relaxation, Eleventh International Conference of Domain Decomposition Methods (C-H. Lai, P. Bjørstad, M. Cross, and O. Widlund, eds.), ddm.org, 1999. MR 1827406

7. , Optimal Schwarz waveform relaxation for the one dimensional wave equation, SIAM Journal of Numerical Analysis 41 (2003), no. 5, 1643-1681. MR.2035001 (2005h:65252)

8. P-L. Lions, On the Schwarz alternating method. I., First International Symposium on Domain Decomposition Methods for Partial Differential Equations (Philadelphia, PA) (R. Glowinski, G. H. Golub, G. A. Meurant, and J. Périaux, eds.), SIAM, 1988, pp. 1-42. MR.972510 (90a:65248)

9. M. Sablé-Tougeron, Régularité microlocale pour des problèmes aux limites non linéaires, Ann. Inst. Fourier 36 (1986), 39-82. MR840713 (88b:35021)

10. H. A. Schwarz, Über einen Grenzübergang durch alternierendes Verfahren, Vierteljahrsschrift der Naturforschenden Gesellschaft in Zürich 15 (1870), 272-286.

11. J. Szeftel, Absorbing boundary conditions for nonlinear partial differential equations, Comput. Methods Appl. Mech. Engrg. 195 (2006), 3760-3775. MR2221773 (2007i:35223)

12. _ A nonlinear approach to absorbing boundary conditions for the semilinear wave equation, Math. Comp. 75 (2006), 565-594. MR2196981 (2007f:35204)

13. G. B. Whitham, Linear and nonlinear waves, Pure and Applied Mathematics (New York), John Wiley \& Sons Inc., New York, 1999, Reprint of the 1974 original, A Wiley-Interscience Publication. MR.1699025 (2000c:35001)

LAGA, Institut Galilée, Université Paris XiII, 93430 Villetaneuse, France

E-mail address: halpern@math.univ-paris13.fr

Department of Mathematics, Princeton University, Fine Hall, Washington Road, Princeton, New Jersey 08544-1000, and C.N.R.S., Mathématiques Appliquées de Bordeaux, Université Bordeaux 1, 351 cours de la Libération, 33405 Talence cedex France

E-mail address: jszeftel@math.princeton.edu 